\title{
Sodium abundances of AGB and RGB stars in Galactic globular clusters
}

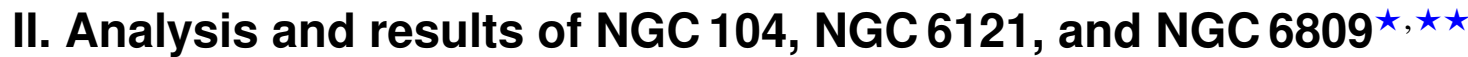

\author{
Y. Wang ${ }^{1,2,3}$, F. Primas ${ }^{3}$, C. Charbonnel ${ }^{4,5}$, M. Van der Swaelmen ${ }^{6}$, G. Bono ${ }^{7,8}$, W. Chantereau ${ }^{4}$, and G. Zhao ${ }^{1,2}$ \\ ${ }^{1}$ Key Laboratory of Optical Astronomy, National Astronomical Observatories, Chinese Academy of Sciences, Beijing 100012 , \\ PR China \\ e-mail: ywang@nao.cas.cn \\ 2 School of Astronomy and Space Science, University of Chinese Academy of Sciences, Beijing 100049, PR China \\ ${ }^{3}$ European Southern Observatory (ESO), Karl-Schwarschild-Str. 2, 85748 Garching b. München, Germany \\ e-mail: fprimas@eso.org \\ ${ }^{4}$ Department of Astronomy, University of Geneva, Chemin des Maillettes 51, 1290 Versoix, Switzerland \\ e-mail: corinne. charbonnel@unige.ch \\ 5 IRAP, UMR 5277 CNRS and Université de Toulouse, 14 Av. E. Belin, 31400 Toulouse, France \\ 6 Institut d'Astronomie et d'Astrophysique, Université Libre de Bruxelles, CP. 226, Boulevard du Triomphe, 1050 Brussels, Belgium \\ 7 Dipartimento di Fisica, Università di Roma Tor Vergata, via della Ricerca Scientifica 1, 00133 Rome, Italy \\ 8 INAF-Osservatorio Astronomico di Roma, via Frascati 33, Monte Porzio Catone, Rome, Italy
}

Received 12 April 2017 / Accepted 24 August 2017

\begin{abstract}
Aims. We investigate the Na abundance distribution of asymptotic giant branch (AGB) stars in Galactic globular clusters (GCs) and its possible dependence on GC global properties, especially age and metallicity.

Methods. We analyze high-resolution spectra of a large sample of AGB and red giant branch (RGB) stars in the Galactic GCs NGC 104, NGC 6121, and NGC 6809 obtained with FLAMES/GIRAFFE at ESO/VLT, and determine their Na abundances. This is the first time that the AGB stars in NGC 6809 are targeted. Moreover, to investigate the dependence of AGB Na abundance dispersion on GC parameters, we compare the AGB $[\mathrm{Na} / \mathrm{H}]$ distributions of a total of nine GCs, with five determined by ourselves with homogeneous method and four from literature, covering a wide range of GC parameters.

Results. NGC 104 and NGC 6809 have comparable AGB and RGB Na abundance distributions revealed by the K-S test, while NGC 6121 shows a lack of very Na-rich AGB stars. By analyzing all nine GCs, we find that the Na abundances and multiple populations of AGB stars form complex picture. In some GCs, AGB stars have similar Na abundances and/or second-population fractions as their RGB counterparts, while some GCs do not have Na-rich second-population AGB stars, and various cases exist between the two extremes. In addition, the fitted relations between fractions of the AGB second population and GC global parameters show that the AGB second-population fraction slightly anticorrelates with GC central concentration, while no robust dependency can be confirmed with other GC parameters.

Conclusions. Current data roughly support the prediction of the fast-rotating massive star (FRMS) scenario. However, considering the weak observational and theoretical trends where scatter and exceptions exist, the fraction of second-population AGB stars can be affected by more than one or two factors, and may even be a result of stochasticity.
\end{abstract}

Key words. stars: abundances - globular clusters: general - globular clusters: individual: NGC $104-$ globular clusters: individual: NGC 6121 - globular clusters: individual: NGC 6809

\section{Introduction}

Galactic globular clusters (GCs) have been the subject of a variety of stellar evolution studies; first, because for a long time they were thought to consist of a single stellar population (i.e., coeval and sharing the same initial chemical properties) thus making them the ideal stellar laboratory, and second, because of their more recently discovered intriguing complexity of being inhabited by multiple stellar populations, a feature that has turned out to be common to most Galactic globular clusters.

\footnotetext{
^ Based on observations made with ESO telescopes at the La Silla Paranal Observatory under programme ID 093.D-0818(A).

$\star \star$ Full Tables 3, 5, and 7 are only available at the CDS via anonymous ftp to cdsarc.u-strasbg. fr (130.79.128.5) or via http://cdsarc.u-strasbg.fr/viz-bin/qcat?J/A+A/607/A135
}

This multiplicity has been identified based on the appearance of multimodal sequences in different regions (e.g., main sequence (MS), sub-giant branch (SGB), red giant branch (RGB) and horizontal branch (HB)) of GC color-magnitude diagrams (CMD; e.g., Piotto et al. 2012; Milone et al. 2012b; Piotto et al. 2015; Milone et al. 2015a,b; Nardiello et al. 2015b) that were associated to the variations in $\mathrm{He}$ and light element (e.g., $\mathrm{C}, \mathrm{N}$, and $\mathrm{O}$ ) abundances in their initial chemical composition (see e.g., Milone et al. 2012b; Chantereau et al. 2015, and references therein).

With the advent of multi-object spectrographs mounted on 8-10 m-class telescopes, detailed chemical abundance analyses have also uncovered specific features - elemental (anti-)correlations - between the light element pairs $\mathrm{C}-\mathrm{N}$, 
O-Na, Mg-Al (e.g., Carretta 2016, for a recent review). These are commonly interpreted as a signature of the existence of at least two stellar populations: a first-population (1P) of GC stars displaying $\mathrm{Na}$ and $\mathrm{O}$ abundances consistent with that of halo field stars of similar metallicity; and a second-population (2P) of GC stars characterized by Na overabundances and $\mathrm{O}$ deficiencies. Although the $\mathrm{O}-\mathrm{Na}$ pair is probably the most documented one in terms of data, a similar picture is also derived from the other pairs, $\mathrm{Mg}-\mathrm{Al}$ (e.g., Carretta et al. 2014; Carretta 2014) and C-N (e.g., Carretta et al. 2005; Pancino et al. 2010).

A wealth of observational data has been collected and analyzed for a respectable number of Galactic GCs at different evolutionary phases, for example, from MS and SGB to RGB and HB (see Wang et al. 2016, hereafter Paper I, for a more detailed summary). However, asymptotic giant branch (AGB) stars have rarely been targeted in a systematic way until very recently, due to their paucity in GCs (a result of their short lifetime) and inefficient RGB/AGB separation criteria. Recently, several studies have focused on GC AGB stars, mainly stimulated by the claim by Campbell et al. (2013, hereafter C13) that no Na-rich 2P AGB stars exists in NGC 6752. This striking finding was challenged by Lapenna et al. (2016) who reobserved the 20 AGB stars of the C13 sample at higher resolution with ESO-VLT/UVES, and found that both $1 \mathrm{P}$ and $2 \mathrm{P}$ stars populate the AGB of NGC 6752, with only stars with extreme $\mathrm{Na}$ enhancement missing. The presence of $2 \mathrm{P}$ AGB stars in this GC is also supported by Gruyters et al. (2017) who claimed a photometric split on the AGB sequence using Strömgrem photometry. Other GC AGB stars have also been scrutinized. Johnson et al. (2015) studied 35 AGB stars in 47 Tuc (NGC 104) and found that the AGB and RGB samples of 47 Tuc have nearly identical $[\mathrm{Na} / \mathrm{Fe}]$ dispersions, with only a small fraction $(\$ 20 \%)$ of Na-rich stars that may fail to ascend the AGB. García-Hernández et al. (2015) showed that 2P AGB stars exist in metal-poor GCs with a study of $\mathrm{Mg}$ and $\mathrm{Al}$ abundances in 44 AGB stars from four metal-poor GCs (M 13, M 5, M 3 and M 2). In Paper I, we looked at NGC 2808 and also found that its AGB and RGB stars share similar Na abundance dispersions. Moreover, we found more Na-rich 2P stars in the AGB sample than in the RGB one. The multiple populations in AGB stars in NGC 2808 was also confirmed by Marino et al. (2017) who carried out a study combining spectroscopy and photometry. They also looked at NGC 6121 (M 4) and found it hosts two main populations in agreement with the finding by Lardo et al. (2017) that AGB stars show broadened distribution in close analogy with their RGB counterparts in the $C_{U B I}{ }^{1}-V$ diagram. We note, however, that their conclusion on NGC 6121 contradicts the result of MacLean et al. (2016) who found that the AGB is populated by $\mathrm{Na}$-poor and O-rich stars (from the analysis of $15 \mathrm{AGB}$ and 106 RGB stars). NGC 6266 (M62) was also found to have only 1P AGB stars by Lapenna et al. (2015), but their conclusion may be affected by the small number statistics of their sample (6 AGB and 13 RGB stars).

It is now largely accepted that GCs experienced selfenrichment during their early evolution, and that $2 \mathrm{P}$ stars formed out of the Na-rich, O-poor ashes of high-temperature-burning hydrogen ejected by more massive $1 \mathrm{P}$ stars and diluted with interstellar gas (e.g., Prantzos \& Charbonnel 2006; Prantzos et al. 2007). However, the nature of the polluters remains highly debated, as well as the mode and timeline of the formation of $2 \mathrm{P}$ stars. Among the most commonly-invoked scenarios, one finds fast-rotating massive stars (FRMS, with initial masses above

$1 C_{U B I}=(U-B)-(B-I)$. $\sim 25 M_{\odot}$; $\quad$ Maeder \& Meynet 2006; Prantzos \& Charbonnel 2006; Decressin et al. 2007a,b; Krause et al. 2013), massive AGB stars (with initial masses of $\sim 6-11 M_{\odot}$; Ventura et al. 2001; D'Ercole et al. 2010; Ventura \& D'Antona 2011; Ventura et al. 2013) and supermassive stars $\left(\sim 10^{4} M_{\odot}\right.$; Denissenkov \& Hartwick 2014; Denissenkov et al. 2015). Other possible polluters have also been explored, like massive stars in close binaries (10-20 $M_{\odot}$; de Mink et al. 2009; Izzard et al. 2013), FRMS paired with AGB stars (Sills \& Glebbeek 2010) or with high-mass interactive binaries (Bastian et al. 2013; Cassisi \& Salaris 2014). So far, none of the proposed scenarios have been able to reconcile all aspects of the formation and evolution of GCs with the spectroscopic and photometric complexity exhibited by these systems, nor with the new constraints coming from extragalactic young massive clusters that have masses similar to the initial mass postulated for GCs within the self-enrichment framework (e.g., Bastian et al. 2015; Renzini et al. 2015; Krause et al. 2016; Charbonnel 2016).

One key feature to pay attention to is how the various scenarios differ from one another. The origin and amount of $\mathrm{He}$ enrichment predicted for $2 \mathrm{P}$ stars is one such example, which has important consequences on the way the various sequences of the CMDs can be populated (e.g., D'Antona et al. 2010; Chantereau et al. 2015, 2016). Interestingly enough, the different theoretical predictions for the coupling between $\mathrm{He}$ and $\mathrm{Na}$ enrichments in the initial composition of $2 \mathrm{P}$ stars are expected to differentially affect the extent of the $\mathrm{Na}$ dispersion today among RGB and AGB stars in individual GCs, in proportions that depend on their age and metallicity (Charbonnel \& Chantereau 2016). In the original FRMS framework, 2P low-mass stars are predicted to be born with large and correlated spreads in both $\mathrm{He}$ and $\mathrm{Na}$ abundances (Decressin et al. 2007a). Since the lifetime and the fate of stars strongly depend on their initial He content, the FRMS scenario predicts that, above a certain threshold, or cutoff, of initial $\mathrm{He}$ and $\mathrm{Na}$ abundance, $2 \mathrm{P}$ stars do miss the AGB (so-called AGB-manqué) and evolve directly towards the white dwarf stage after central He burning (Charbonnel et al. 2013; Chantereau et al. 2015). This provides in principle a nice explanation for the lack of Na-rich AGB stars observed in NGC 6752 by C13. Charbonnel \& Chantereau (2016) have also shown that within the original FRMS scenario, the maximum Na content expected on the AGB is a (weak) function of both the metallicity and the age of GCs. Namely, at a given metallicity, younger clusters are expected to host AGB stars exhibiting a larger $\mathrm{Na}$ spread than older clusters, and at a given age, higher $\mathrm{Na}$ dispersion along the AGB is predicted in metal-poor GCs than in the metal-rich ones. Additionally, mass loss along the RGB has been shown to strongly impact the evolution of low-mass stars on the AGB, and therefore to modify the theoretical Na cut on the AGB (Charbonnel \& Chantereau 2016; see also Cassisi et al. 2014): the higher the mass loss, the stronger the trends with age and metallicity. However, the situation might be much more complex, as revealed by the derivation of the helium variations between 1P and 2P stars in several GCs by multiwavelength photometry of multiple sequences, which turn out to be much lower than predicted by both the original FRMS and AGB scenario (Anderson et al. 2009; di Criscienzo et al. 2010; Pasquini et al. 2011; Milone et al. 2012a,b, 2013, 2015b; Piotto et al. 2013; Marino et al. 2014; Larsen et al. 2015; Nardiello et al. 2015a,b). It is therefore fundamental to test model predictions with observations of AGB and RGB stars in GCs spanning a large range in age and metal content. This is necessary to probe the degree of stochasticity lying behind the broad variety of chemical patterns observed in GCs (e.g., Bastian et al. 2015). 
Considering the current limited sample of GC AGB stars with accurately determined $\mathrm{Na}$ (and $\mathrm{O}$ ) abundances, we carried out a systematic observational campaign of four GCs (NGC 104, NGC 2808, NGC 6121 and NGC 6809). We have already presented our first results of NGC 2808 in Paper I. Here, we report and discuss our results of the other three GCs, investigating whether the presence of Na-rich stars on AGB is dependent on metallicity and/or other GC parameters.

The paper is organized as follows. In Sects. 2 and 3, we describe the observations and detail the analysis of the data for our sample of GCs. In Sect. 4, we present the re-analysis of C13 data for NGC 6752 and we show other four GCs from the literature. In Sect. 5, we compare the behavior of $\mathrm{Na}$ along the AGB and the RGB for the full GC sample (ours plus literature); we discuss also the possible correlations between the corresponding fractions of $1 \mathrm{P}$ and $2 \mathrm{P}$ stars and the GC global properties, and compare with the theoretical predictions of the original FRMS scenario. A summary and concluding remarks close the paper in Sect. 6.

\section{Observation and data reduction}

As already mentioned in Paper I, we selected our targets in NGC 104, NGC 6121, and NGC 6809 from the Johnson-Morgan photometric database which is part of the project described in Stetson $(2000,2005)$ and covers a magnitude range of about three magnitudes for each GC. To distinguish AGB from RGB stars, we used several CMDs with different combinations of color indices and magnitudes. We found that in the CMDs of, for example, $(U-I)-U,(U-I)-I$, and $(B-I)-V$, AGB and RGB stars can be separated efficiently, similarly to García-Hernández et al. (2015). Figure 1 shows the location of the member stars in the CMDs of $(B-I)-V$.

All our spectra were obtained with the high-resolution multi-object spectrograph FLAMES, mounted on ESO/VLTUT2 (Pasquini et al. 2003), taking advantage of GIRAFFE (HR 13, HR 15, and HR 19) for the majority of our sample stars and used the UVES fibres (Red 580) for the brightest objects of each cluster. Table 1 summarizes the most relevant details of our observational campaign. Data reduction followed standard procedures and was carried out as described in Paper I. The final co-added spectra have signal-to-noise ratios $(\mathrm{S} / \mathrm{N})$ ranging between 50 and 400 for the GIRAFFE spectra and 50 and 230 for the UVES spectra, depending on the magnitude of the star. We identified non-cluster-member stars based on the derived stellar radial velocities and removed them from further analysis. The number of stars (initially observed and later confirmed as cluster members) are listed in Table 2 for each of the three GCs, together with their derived mean barycentric radial velocities, whereas Table 3 lists the most relevant information of only the member stars, that is, their evolutionary phases (AGB/RGB), the instrument used for collecting the spectrum (GIRAFFE/UVES), and their coordinates, photometry and barycentric radial velocities.

\section{Stellar parameters and abundance analysis of our sample stars}

\subsection{Effective temperature and surface gravity}

Optical $B, V$, and $I$ magnitudes are available for all our stars from the photometric database mentioned in Sect. 2. By crossmatching the coordinates of our targets with the 2MASS catalog (Skrutskie et al. 2006) we have also compiled the corresponding $J, H$, and $K$ infrared magnitudes.
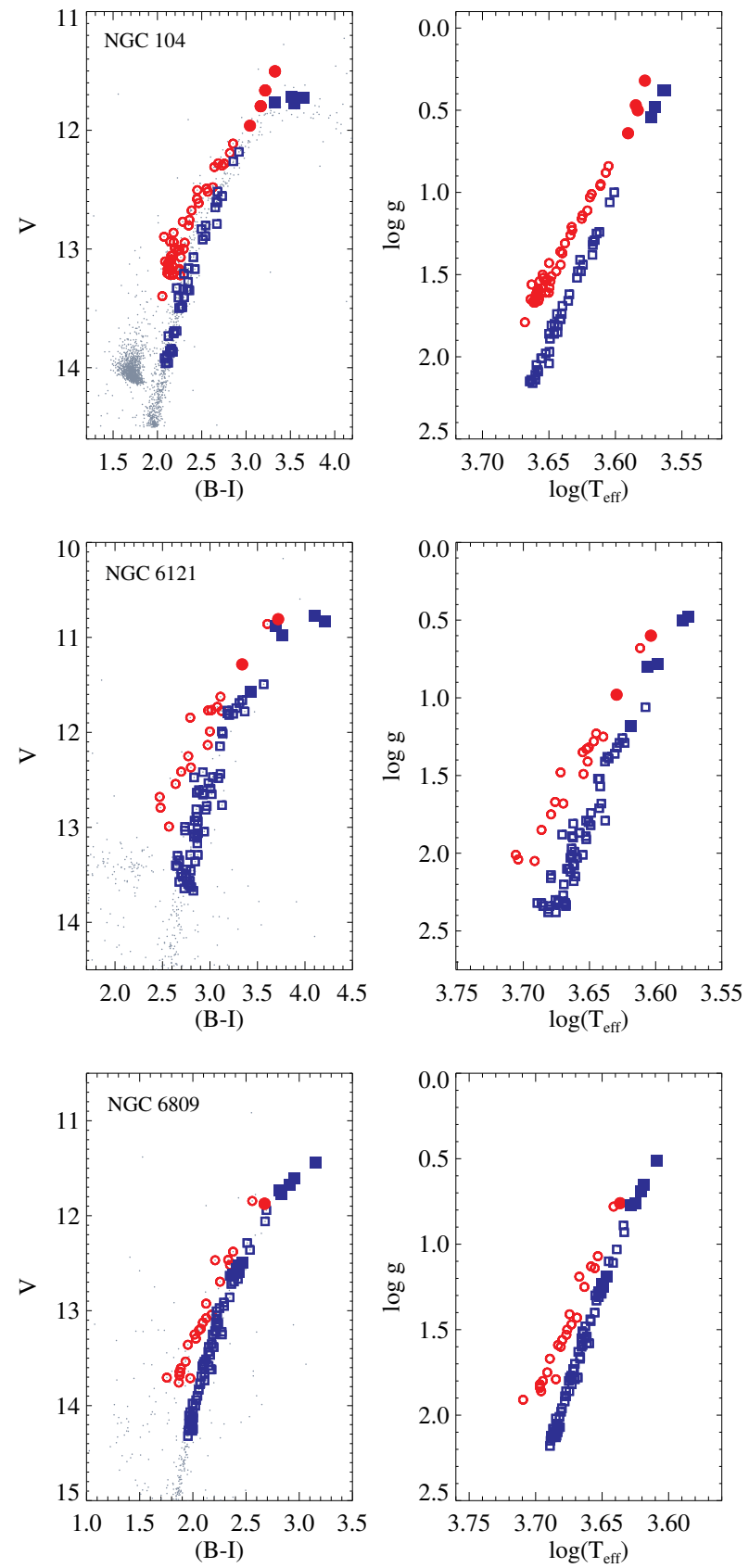

Fig. 1. Photometric CMDs (left) and $\log g-\log T_{\text {eff }}$ distributions (right) of the cluster member stars. Red circles and blue squares represent AGB and RGB stars, respectively, while the GIRAFFE and UVES samples can be distinguished by open and filled symbols, respectively. The same symbols are used throughout the paper.

The foreground reddenings and distance moduli adopted for the three GCs are listed in Table 4. We note that for the distance modulus of NGC 104, Bono et al. (2008) took an average of the values derived from the tip of the red giant branch and the RR Lyrae methods. For NGC 6809, by combining information from photometry ( $V$ magnitude and $B-V$ ) and period data of 13 RR Lyrae stars (Olech et al. 1999), we computed the absolute distance modulus using the dual band metal-dependent PeriodWesenheit (PWZ) relation recently derived by Marconi et al. (2015), which is almost metallicity independent in B and V. For NGC 104 and NGC 6809, we adopted the Cardelli et al. (1989) 
Table 1. Summary of the observations.

\begin{tabular}{ccccccc}
\hline \hline Instrument & Setup & $R$ & $\begin{array}{c}\lambda \text {-range } \\
(\mathrm{nm})\end{array}$ & $\begin{array}{c}\text { Exp. time (s) } \\
\text { NGC 104 (47 Tuc) }\end{array}$ & $\begin{array}{c}\text { Exp. time (s) } \\
\text { NGC 6121 (M 4) }\end{array}$ & $\begin{array}{c}\text { Exp. time (s) } \\
\text { NGC 6809 (M 55) }\end{array}$ \\
\hline GIRAFFE & HR 13 & 22500 & $612.0-640.5$ & $2 \times 1200$ & $1 \times 2700$ & $2 \times 3600$ \\
& HR 15 & 19300 & $660.7-696.5$ & $2 \times 1200$ & $1 \times 1800$ & $2 \times 2770$ \\
& HR 19 & 14000 & $774.5-833.5$ & $2 \times 2700$ & $2 \times 2700$ & $2 \times 2770$ \\
UVES-fibre & Red 580 & 47000 & $480-680$ & $2 \times 3600+4 \times 1200$ & $3 \times 2700+1 \times 1800$ & $2 \times 3600+4 \times 2770$ \\
\hline
\end{tabular}

Table 2. Number of stars (observed and confirmed cluster members) and derived barycentric radial velocities.

\begin{tabular}{ccccccc}
\hline \hline GC & Observed stars & $\begin{array}{c}\text { AGB star } \\
\text { members }\end{array}$ & $\begin{array}{c}\text { RGB star } \\
\text { members }\end{array}$ & $\begin{array}{c}\text { RV } \\
\left(\mathrm{km} \mathrm{s}^{-1}\right)\end{array}$ & $\begin{array}{c}\sigma_{\mathrm{RV}} \\
\left(\mathrm{km} \mathrm{s}^{-1}\right)\end{array}$ & $\begin{array}{c}\mathrm{RV}_{\text {Harris }} \\
\left(\mathrm{km} \mathrm{s}^{-1}\right)\end{array}$ \\
\hline NGC 104 & 94 & $46+4$ & $40+4$ & $-1-7.3$ & 9.9 & -18.0 \\
NGC 6121 & 95 & $17+2$ & $63+5$ & 70.4 & 3.3 & 70.7 \\
NGC 6809 & 110 & $23+1(1)$ & $74+10(6)$ & 173.6 & 3.7 & 174.7 \\
NGC 2808 & 100 & $30+3$ & $38+2$ & 104.6 & 8.0 & 101.6 \\
\hline
\end{tabular}

Notes. Numbers provided in 3th and 4th columns are given in the format of stars observed with "GIRAFFE + UVES", with the number of stars in common specified between parentheses. Our data on NGC 2808 from Paper I are also reported to help in the comparison.

relations ${ }^{2}$, as in Paper I. NGC 6121, instead, is more peculiar because it is located in the Galactic plane behind the Sco-Oph cloud complex, and a non-standard reddening law should be applied (Hendricks et al. 2012, and references therein). For this GC, we thus adopted the recent reddening law and distance modulus reported by Hendricks et al. (2012) who used a combination of broadband near-infrared and optical Johnson-Cousins photometry to study the dust properties in the line of sight to this cluster.

As in Paper I, we derived the stellar effective temperatures ( $\left.T_{\text {eff }}\right)$ using the Ramírez \& Meléndez (2005) photometric calibrations for giants and adopting five de-reddened color indices, that is, $(B-V)_{0},(V-I)_{0},(V-J)_{0},(V-H)_{0}$, and $(V-K)_{0}$. The variations among these temperature scales are smaller than or comparable to the error of the mean temperature of the five scales. So we took the mean value of the temperatures derived from the five color indices as our final $T_{\text {eff }}$. The surface gravities $\log g$ were derived from first principles, that is, by using effective temperatures, bolometric corrections (taken from Alonso et al. 1999) and stellar masses. For the latter, test runs with our stellar evolution code showed that stellar masses on the RGB have a slight dependence on the metallicity and age of the cluster, while these differences become negligible on the AGB. Therefore, we assumed $m=0.61 M_{\odot}$ for the AGB stars in all three clusters, whereas we differentiated the values for the RGB stars with $m_{\mathrm{RGB}, \mathrm{NGC104}}=0.91 M_{\odot}, m_{\mathrm{RGB}, \mathrm{NGC} 6121}=0.87 M_{\odot}$ and $m_{\mathrm{RGB}, \mathrm{NGC6809}}=0.81 M_{\odot}$. The right panels of Fig. 1 show the $\log T_{\text {eff }}-\log g$ distributions of the member stars.

It is worth mentioning that all three GCs suffer from some differential reddening. In the case of NGC 104 and NGC 6809 the differential reddenings are small and comparable in magnitude to the errors on the derived reddenings (cf. Table 4 and references therein). If they are taken into account as one extra source of uncertainty, the typical errors on their final effective temperatures and gravities become of the order of $\pm 70 \mathrm{~K}$ (NGC 104), $\pm 80 \mathrm{~K}$ (NGC 6809), and \pm 0.06 (for both clusters), respectively. The case of NGC 6121 is however more complex because of its much larger differential reddening ( $\sim 0.20 \mathrm{mag}$, Hendricks et al. 2012). The errors on $T_{\text {eff }}$ and $\log g$

$2 A(B)=4.145 E(B-V) ; A(V)=3.1 E(B-V) ; A(I)=1.485 E(B-V)$; $A(J)=0.874 E(B-V) ; A(H)=0.589 E(B-V) ; A(K)=0.353 E(B-V)$. could reach $\Delta T_{\text {eff }} \sim \pm 260 \mathrm{~K}$ and $\Delta \log g \sim \pm 0.12$ dex if the reported differential reddening is taken into account at face value, a significant difference from $\Delta T_{\text {eff }} \sim \pm 50 \mathrm{~K}$ and $\Delta \log g \sim$ \pm 0.03 dex derived by accounting for only the intrinsic error on the reddening. However, a range of differential reddening values has been proposed for this cluster, for example, from $\sim 0.05 \mathrm{mag}$ by Cudworth \& Rees (1990) and $\sim 0.10 \mathrm{mag}$ by Monelli et al. (2013), Lardo et al. (2017) up to $\sim 0.25 \mathrm{mag}$ by Mucciarelli et al. (2011). Considering the complexity and uncertainties in the reddening of NGC 6121, we empirically took the median and decided to consider $\Delta T_{\text {eff }} \sim \pm 150 \mathrm{~K}$ and $\Delta \log g \sim \pm 0.08$ dex as representative of our analytical uncertainties.

\subsection{Metallicity and microturbulent velocity}

Metallicity $([\mathrm{Fe} / \mathrm{H}])$ and microturbulence $\xi_{\mathrm{t}}$ were determined as in Paper I, where a detailed description of our methodology is provided. In short, metallicities were derived by measuring the equivalent widths (EWs) of both Fe I and Fe II unblended lines, restricting our selection to lines with EWs between $20 \mathrm{~m} \AA$ and $120 \mathrm{~mA}$. For the computation of the abundances, we used 1D LTE spherical MARCS model atmospheres (Gustafsson et al. 2008), the LTE stellar line analysis programme MOOG (Sneden 1973, 2014 version) and we assumed a solar iron abundance of $\log \epsilon(\mathrm{Fe})_{\odot}=7.50$ (Asplund et al. 2009). Because of their known inter-dependencies, all stellar parameters $\left(T_{\text {eff }}, \log g,[\mathrm{Fe} / \mathrm{H}]\right.$, $\xi_{\mathrm{t}}$ ) were derived iteratively and following standard procedures. Since standard LTE analyses of Fe I lines tend to underestimate the true iron abundance, we applied non-LTE (NLTE) corrections to all our LTE Fe I values (Lind et al. 2012, and references therein).

Our final stellar parameters are summarized in Table 5, while Table 6 lists the mean metallicities of the AGB and RGB samples in the three GCs. For convenience, we have added to this table also the values derived in Paper I for NGC 2808. We note that our RGB results agree well with those derived by Carretta et al. (2009) within the associated errors (cf. two rightmost columns of Table 6). A more detailed comparison with the literature forms part of Sect. 4.

Finally, as our overall metallicity of each GC, we chose to use the average value of $[\mathrm{Fe} \mathrm{I} / \mathrm{H}]_{\mathrm{NLTE}}$ and $[\mathrm{Fe} \mathrm{II} / \mathrm{H}]$, that is, 
Y. Wang et al.: Sodium abundances of AGB and RGB stars in Galactic globular clusters. II.

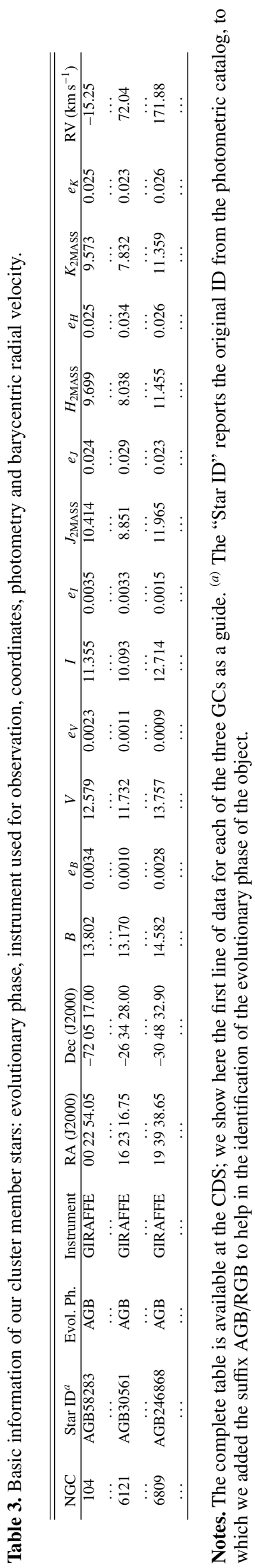

Table 4. Foreground reddenings, differential reddenings and distance moduli of sample GCs.

\begin{tabular}{cccc}
\hline \hline GC & $E(B-V)$ & $\delta E(B-V)$ & $(m-M)_{V}$ \\
\hline NGC 104 & $0.04^{a}$ & $0.028^{d}$ & $13.40^{a}$ \\
NGC 6121 & $0.37^{b}$ & $0.200^{b}$ & $11.28^{b}$ \\
NGC 6809 & $0.11^{c}$ & $0.027^{d}$ & 13.61 \\
\hline
\end{tabular}

References. (a) Bono et al. (2008); (b) Hendricks et al. (2012); ${ }^{(c)}$ Richter et al. (1999); ${ }^{(d)}$ Bonatto et al. (2013).

$[\mathrm{Fe} / \mathrm{H}]_{\mathrm{NGC} 104}=-0.82 \mathrm{dex},[\mathrm{Fe} / \mathrm{H}]_{\mathrm{NGC} 6121}=-1.14 \mathrm{dex}$, and $[\mathrm{Fe} / \mathrm{H}]_{\mathrm{NGC} 6809}=-1.86$ dex.

\subsection{Sodium abundance}

Our stellar Na abundances were derived via spectrum synthesis of the Na doublet at $6154-6160 \AA$, using MOOG and MARCS spherical model atmospheres interpolated to match our derived stellar parameters. A solar sodium abundance of $\log \epsilon(\mathrm{Na})_{\odot}=$ 6.24 (Asplund et al. 2009) was adopted throughout the analysis. As already done for NGC 2808 (cf. Paper I) we took the average of the abundances derived from both doublet lines as our final $\mathrm{Na}$ abundance. Similarly to iron, $\mathrm{Na}$ abundances determined from neutral lines (as in our case) are also affected by the NLTE effect and were therefore corrected accordingly.

We list the $\mathrm{Na}$ abundances derived for the individual stars in Table 7, along with the NLTE-corrected values based on the grids computed by Lind et al. (2011). Table 8 is similar to Table 6, now summarizing the average $\mathrm{Na}$ abundances of our three GCs. For convenience, it also reports the results from our previous analysis on NGC 2808.

We note that in the two most metal-rich GCs in our sample, the Na doublet was saturated or approaching saturation in 27 stars of NGC 104 and 4 stars of NGC 6121, respectively. These stars were dropped from any further discussion.

\subsection{Error analysis}

We estimated the uncertainties in our derived abundances, following the procedures described in Paper I and considering errors of both random and systematic nature.

As random measurement uncertainty, we considered $\sigma / \sqrt{\mathrm{N}}$, where $\sigma$ is the line-to-line dispersion and $N$ is the number of lines measured. A correction according to a t-distribution was applied to the $\mathrm{Na}$ and $\mathrm{Fe}$ II abundances considering the limited number of lines present in our spectra (cf. Paper I for more details).

For the systematic uncertainty, we selected a total of six stars per GC: Four stars observed with GIRAFFE - one cool/hot in each AGB/RGB sample; and two stars observed with UVESfibre - one cool AGB and one cool RGB, as this sub-sample includes only cool stars. With these 18 stars, we tested the effect of varying stellar parameters and EWs (or other key parameters of the analysis) by their associated errors on the derived abundances.

Typical total uncertainties, combining both random and systematic sources of error, are summarized in Table 9 for the GIRAFFE and UVES samples of each GC. 
Table 5. Stellar parameters of our sample stars.

\begin{tabular}{|c|c|c|c|c|c|c|c|c|c|c|c|}
\hline$\overline{\mathrm{NGC}}$ & Star ID & Evol. Ph. & $\begin{array}{l}T_{\text {eff }} \\
(\mathrm{K})\end{array}$ & $\begin{array}{c}\sigma T_{\text {eff }}{ }^{a} \\
(\mathrm{~K})\end{array}$ & $\overline{l o g} g$ & $\begin{array}{c}\xi_{\mathrm{t}} \\
\left(\mathrm{km} \mathrm{s}^{-1}\right)\end{array}$ & $\begin{array}{c}{[\mathrm{Fe} \mathrm{I} / \mathrm{H}]_{\mathrm{LTE}}} \\
(\mathrm{dex})\end{array}$ & $\begin{array}{c}\text { rms_lines } \\
\text { (dex) }\end{array}$ & $\begin{array}{c}{[\mathrm{Fe} \mathrm{II} / \mathrm{H}]} \\
(\mathrm{dex})\end{array}$ & $\begin{array}{c}\text { rms_lines } \\
\text { (dex) }\end{array}$ & $\begin{array}{c}{[\mathrm{Fe} \mathrm{I} / \mathrm{H}]_{\mathrm{NLTE}}} \\
(\mathrm{dex})\end{array}$ \\
\hline 104 & AGB58283 & AGB & 4291 & 39.3 & 1.23 & 1.60 & -0.85 & 0.12 & -0.89 & 0.15 & -0.82 \\
\hline$\ldots 121$ & $\begin{array}{c}\ldots \\
\text { AGB30561 }\end{array}$ & $\ldots$ & $\ldots$ & 25.3 & $\begin{array}{l}\ldots \\
1.28\end{array}$ & $\begin{array}{l}\ldots \\
1.75\end{array}$ & $\begin{array}{c}\ldots \\
-1.29\end{array}$ & 0.10 & $\begin{array}{c}\cdots \\
-1.26\end{array}$ & 0.02 & $\begin{array}{c}\cdots \\
-1.24\end{array}$ \\
\hline 6809 & AGB246868 & AGB & 4964 & 25.7 & 1.86 & 1.16 & -2.00 & 0.15 & -1.89 & 0.01 & -1.91 \\
\hline$\ldots$ & $\ldots$ & $\ldots$ & $\ldots$ & $\ldots$ & $\ldots$ & $\ldots$ & $\ldots$ & $\ldots$ & $\ldots$ & $\ldots$ & $\ldots$ \\
\hline
\end{tabular}

Notes. The complete table is available at the CDS; we show here the first line of data for each GC as a guide. ${ }^{(a)} \sigma T_{\text {eff }}$ is the scatter of the temperatures derived from the five colors we considered.

Table 6. Iron abundances of AGB and RGB samples of GCs.

\begin{tabular}{|c|c|c|c|c|c|c|c|}
\hline GC/sample & $\begin{array}{l}\mathrm{Fe} / / \mathrm{H}]_{\text {LTE }} \\
\quad(\mathrm{dex})\end{array}$ & $\begin{array}{l}{[\mathrm{Fe} \mathrm{I} / \mathrm{H}]_{\mathrm{NLTE}}} \\
(\mathrm{dex})\end{array}$ & $\begin{array}{c}\operatorname{rms}\left([\mathrm{Fe} \mathrm{I} / \mathrm{H}]_{\text {NLTE }}\right) \\
(\mathrm{dex})\end{array}$ & $\begin{array}{c}\text { Fe II/H }] \\
(\operatorname{dex})\end{array}$ & $\begin{array}{l}\operatorname{rms}([\mathrm{Fe} I \mathrm{II} / \mathrm{H}]) \\
(\mathrm{dex})\end{array}$ & $\begin{array}{c}{[\mathrm{Fe} \mathrm{I} / \mathrm{H}]_{\text {LTE }}(\text { Carretta })} \\
(\mathrm{dex})\end{array}$ & $\begin{array}{c}{[\mathrm{Fe} \mathrm{II} / \mathrm{H}](\text { Carretta) }} \\
(\mathrm{dex})\end{array}$ \\
\hline NGC 104 & & & & & & & \\
\hline AGB sample & -0.82 & -0.79 & 0.07 & -0.91 & 0.13 & & \\
\hline RGB sample & -0.77 & -0.75 & 0.08 & -0.82 & 0.15 & $-0.743 \pm 0.047$ & $-0.769 \pm 0.081$ \\
\hline NGC 6121 & & & & & & & \\
\hline AGB sample & -1.21 & -1.15 & 0.08 & -1.22 & 0.09 & & \\
\hline RGB sample & -1.15 & -1.11 & 0.08 & -1.15 & 0.06 & $-1.200 \pm 0.043$ & $-1.197 \pm 0.082$ \\
\hline NGC 6809 & & & & & & & \\
\hline AGB sample & -2.03 & -1.93 & 0.07 & -1.91 & 0.04 & & \\
\hline RGB sample & -1.92 & -1.85 & 0.06 & -1.84 & 0.06 & $-1.967 \pm 0.041$ & $-1.933 \pm 0.093$ \\
\hline NGC 2808 & & & & & & & \\
\hline AGB sample & -1.19 & -1.14 & 0.09 & -1.14 & 0.10 & & \\
\hline RGB sample & -1.12 & -1.08 & 0.07 & -1.09 & 0.07 & $-1.100 \pm 0.059$ & $-1.160 \pm 0.089$ \\
\hline
\end{tabular}

Notes. For convenience, the last rows of the table refer to our results on NGC 2808 from Paper I. The right-most two columns list the metallicities derived from RGB stars by Carretta et al. (2009) for NGC 104, NGC 6121, and NGC 6809, with the associated errors being the total star-to-star errors in their Table A.3, while the data for NGC 2808 are from Carretta et al. (2006).

Table 7. Na abundances of our sample stars.

\begin{tabular}{cccccc}
\hline \hline NGC & Star ID & Evol. Ph. & $\begin{array}{c}{[\mathrm{Na} / \mathrm{H}]_{\text {LTE }}} \\
(\mathrm{dex})\end{array}$ & $\begin{array}{c}{[\mathrm{Na} / \mathrm{H}]_{\mathrm{NLTE}}} \\
(\mathrm{dex})\end{array}$ & $\begin{array}{c}{[\mathrm{Na} / \mathrm{Fe}]_{\mathrm{NLTE}}} \\
(\mathrm{dex})\end{array}$ \\
\hline 104 & AGB58283 & AGB & -0.10 & -0.21 & 0.61 \\
$\ldots$ & $\ldots$ & $\ldots$ & $\ldots$ & $\ldots$ & $\ldots$ \\
6121 & AGB30561 & AGB & -0.79 & -0.85 & 0.39 \\
$\ldots$ & $\ldots$ & $\ldots$ & $\ldots$ & $\ldots$ & $\ldots$ \\
6809 & AGB246868 & AGB & -1.45 & -1.53 & 0.38 \\
$\ldots$ & $\ldots$ & $\ldots$ & $\ldots$ & $\ldots$ & $\ldots$ \\
\hline
\end{tabular}

Notes. The complete table is available at the CDS; we show here the first line of data for each GC as a guide.

\subsection{Stars observed with both GIRAFFE and UVES-fibre}

In the case of NGC 6809, we were able to optimize our fibre configurations by swapping targets between fibre types. We thus observed seven objects (one AGB and six RGB) with both GIRAFFE-Medusa and UVES-fibre, which allowed us to test whether or not any zero-point abundance difference exists between the two different sets of spectra. We found the following average differences (UVES-fibre minus GIRAFFE): $\Delta[\mathrm{Fe} \mathrm{I} / \mathrm{H}]_{\mathrm{NLTE}}=+0.02 \pm 0.04 \mathrm{dex}, \Delta[\mathrm{Fe} \mathrm{II} / \mathrm{H}]=$ $-0.03 \pm 0.07 \mathrm{dex}, \Delta[\mathrm{Na} / \mathrm{H}]_{\mathrm{NLTE}}=-0.01 \pm 0.05 \mathrm{dex}$, and $\Delta[\mathrm{Na} / \mathrm{Fe}]_{\mathrm{NLTE}}=-0.03 \pm 0.06 \mathrm{dex}$. These results show that, despite a lower resolution and smaller spectral coverage, the majority of our spectra (indeed observed with GIRAFFE) match very well the results obtained from the analysis of the UVESfibre counterparts. We highlight that in the following Sections, although it does not make any significant difference, we have considered the UVES-fibre results for these seven stars.

\subsection{Observed $\mathrm{Na}$ abundance distribution along the $R G B$ and $A G B$}

Our final $\mathrm{Na}$ abundance distributions are shown in Fig. 2 as a function of effective temperature for $\mathrm{AGB}$ and RGB stars in NGC 104, NGC 6121, and NGC 6809. Here, we present them in the form of NLTE $[\mathrm{Na} / \mathrm{H}]$ and $[\mathrm{Na} / \mathrm{Fe}]$ (where Fe now only refers to $\mathrm{Fe}$ I) ratios, as listed in Table 7 . However, as pointed out by Campbell et al. (2017), the $[\mathrm{Fe} \mathrm{I} / \mathrm{H}]$ can be affected by the $T_{\text {eff }}$ scale, which further influences the degree of Fe I-Fe II discrepancy that has been found especially in AGB stars by several works (Ivans et al. 2001; Lapenna et al. 2014, 2015, 2016; Mucciarelli et al. 2015) and also by us (see also Sect. 4.1). Any uncertainty in the determination of the iron abundance will affect the accuracy of the $[\mathrm{Na} / \mathrm{Fe}]$ ratios, while $[\mathrm{Na} / \mathrm{H}]$ is very robust, as also detailed by Campbell et al. (2017) who show that parameter variations between studies (caused by using different methods, tools, input data, etc.) have little effect on the derived 
Y. Wang et al.: Sodium abundances of AGB and RGB stars in Galactic globular clusters. II.

Table 8. Mean Na abundances of AGB and RGB samples in our three new GCs.

\begin{tabular}{cccccccc}
\hline \hline GC & $\begin{array}{c}{[\mathrm{Na} / \mathrm{H}]_{\text {LTE }}} \\
(\mathrm{dex})\end{array}$ & $\begin{array}{c}\delta \mathrm{NLTE} \\
(\mathrm{dex})\end{array}$ & $\begin{array}{c}{[\mathrm{Na} / \mathrm{H}]_{\mathrm{NLTE}}} \\
(\mathrm{dex})\end{array}$ & $\begin{array}{c}\sigma_{[\mathrm{Na} / \mathrm{H}]} \\
(\mathrm{dex})\end{array}$ & $\begin{array}{c}{[\mathrm{Na} / \mathrm{Fe} \mathrm{I}]_{\mathrm{NLTE}}} \\
(\mathrm{dex})\end{array}$ & $\begin{array}{c}\left.\sigma_{[\mathrm{Na} / \mathrm{Fe}]}\right] \\
(\mathrm{dex})\end{array}$ & $\begin{array}{c}\mathrm{IQR}_{[\mathrm{Na} / \mathrm{H}]} \\
(\mathrm{dex})\end{array}$ \\
\hline NGC 104 & & & & & & & \\
AGB sample & -0.42 & -0.07 & -0.49 & 0.19 & 0.30 & 0.20 & 0.285 \\
RGB sample & -0.30 & -0.09 & -0.39 & 0.17 & 0.36 & 0.19 & 0.330 \\
\hline NGC 6121 & & & & & & & \\
AGB sample & -0.90 & -0.07 & -0.97 & 0.17 & 0.19 & 0.16 & 0.300 \\
RGB sample & -0.75 & -0.08 & -0.82 & 0.20 & 0.29 & 0.19 & 0.250 \\
\hline NGC 6809 & & & & & & & \\
AGB sample & -1.60 & -0.07 & -1.66 & 0.13 & 0.27 & 0.15 & 0.220 \\
RGB sample & -1.56 & -0.07 & -1.62 & 0.17 & 0.23 & 0.16 & 0.250 \\
\hline NGC 2808 & & & & & & & \\
AGB sample & -1.00 & -0.06 & -1.06 & 0.13 & 0.09 & 0.16 & 0.160 \\
RGB sample & -0.98 & -0.06 & -1.04 & 0.21 & 0.05 & 0.20 & 0.390 \\
\hline
\end{tabular}

Notes. $\delta$ NLTE is the NLTE correction of the Na abundance. $\sigma$ represents the dispersion (standard deviation) of the corresponding Na abundance. $I Q R_{[\mathrm{Na} / \mathrm{H}]}$ is the inter-quartile range of $[\mathrm{Na} / \mathrm{H}]_{\mathrm{NLTE}}$. For convenience, the last section of the table reports our results on NGC 2808 from Paper I.

Table 9. Total uncertainties of derived $\mathrm{Fe}$ and $\mathrm{Na}$ abundances.

\begin{tabular}{ccccc}
\hline \hline & $\begin{array}{c}{[\mathrm{Fe} \mathrm{I} / \mathrm{H}]} \\
(\mathrm{dex})\end{array}$ & $\begin{array}{c}{[\mathrm{Fe} \mathrm{II} / \mathrm{H}]} \\
(\mathrm{dex})\end{array}$ & $\begin{array}{c}{[\mathrm{Na} / \mathrm{H}]} \\
(\mathrm{dex})\end{array}$ & $\begin{array}{c}{[\mathrm{Na} / \mathrm{Fe} \mathrm{I}]} \\
(\mathrm{dex})\end{array}$ \\
\hline NGC 104 & & & & \\
GIRAFFE sample & \pm 0.07 & \pm 0.12 & \pm 0.13 & \pm 0.13 \\
UVES sample & \pm 0.07 & \pm 0.13 & - & - \\
\hline NGC 6121 & & & & \\
GIRAFFE sample & \pm 0.13 & \pm 0.14 & \pm 0.16 & \pm 0.11 \\
UVES sample & \pm 0.09 & \pm 0.18 & \pm 0.16 & \pm 0.10 \\
\hline NGC 6809 & & & & \\
GIRAFFE sample & \pm 0.11 & \pm 0.07 & \pm 0.13 & \pm 0.12 \\
UVES sample & \pm 0.09 & \pm 0.05 & \pm 0.13 & \pm 0.10 \\
\hline
\end{tabular}

$[\mathrm{Na} / \mathrm{H}]$. This was also our conclusion from Paper I, which led us to base our discussion of the Na distribution in the RGB and AGB samples on the $[\mathrm{Na} / \mathrm{H}]$ abundance indicator only.

For each individual cluster we perform a two-sample Kolmogorov-Smirnov (K-S) test to estimate the similarity of the $[\mathrm{Na} / \mathrm{H}]$ distributions in the AGB and RGB samples. All numbers and conclusions discussed below are roughly confirmed by the dispersions $(\sigma)$ and the interquartile range (IQR) values of the distributions reported in Table 8. For each cluster, we also provide a critical summary of how our derived $\mathrm{Na}$ abundances compare to other abundance studies of similar data quality.

\subsubsection{NGC 104}

NGC 104 (40 AGB, 27 RGB) is the GC in which the AGB and RGB samples are the closest in terms of $\mathrm{Na}$ abundance distributions, with the AGB stars spanning an even slightly larger range of $\mathrm{Na}$ abundances. The K-S test, with $D=0.294$ and $p$ value $=0.101$ derived, indicates that the AGB and RGB samples share the same $[\mathrm{Na} / \mathrm{H}]$ distribution at $95 \%$ significance level.

Our result for this cluster is in very good agreement with Johnson et al. (2015) who found nearly identical [Na/Fe] dispersion in their AGB sample (35 stars) to that in the RGB sample (113 stars) analyzed by Cordero et al. (2014), following the same methodology of Johnson et al. (2015). We carried out a detailed check for 12 AGB and 13 RGB stars in common with our sample (identified by coordinates cross-matching with angular distance $<0.3^{\prime \prime}$ ) and found mean differences (in the sense of our result minus theirs) of $-78 \pm 60 \mathrm{~K}$ in $T_{\text {eff }},-0.07 \pm 0.22$ in $\log g,-0.25 \pm 0.17 \mathrm{~km} \mathrm{~s}^{-1}$ in $\xi_{\mathrm{t}}$, and $-0.12 \pm 0.10 \mathrm{dex}$ in $[\mathrm{Fe} / \mathrm{H}]$. We assign these differences mainly to the different methods employed to derive the stellar parameters $T_{\text {eff }}$ and $\log g$ (photometry in the present study, spectroscopy in Johnson et al. 2015; and Cordero et al. 2014). However, the negligible difference in $[\mathrm{Na} / \mathrm{H}](0.02 \pm 0.12 \mathrm{dex}$; after having checked that their conclusions for $[\mathrm{Na} / \mathrm{Fe}]$ hold also for the $[\mathrm{Na} / \mathrm{H}]$ ratio) confirms the agreement found in the $\mathrm{Na}$ abundance distribution.

\subsubsection{NGC 6121}

We find that in NGC 6121 (19 AGB, 63 RGB), the AGB stars occupy the bottom two-thirds of the $[\mathrm{Na} / \mathrm{H}]$ distribution of the RGB ones. There is an actual difference of 0.26 dex between the maximum $[\mathrm{Na} / \mathrm{H}]$ values of the AGB and RGB samples, indicating a lack of very Na-rich AGB stars in this cluster. The $D=0.408$ and $p$-value $=0.011$ from the K-S test confirms that the two populations do not share the same $\mathrm{Na}$ distribution.

Our results for this GC agree with those of MacLean et al. (2016) who studied a sample of 106 RGB and 15 AGB stars in NGC 6121. In their analysis, they derived a difference of 0.4 dex between the maximum $[\mathrm{Na} / \mathrm{H}]$ values of their $\mathrm{AGB}$ and $\mathrm{RGB}$ samples (compared to our value of $0.26 \mathrm{dex}$, we have more $\mathrm{Na}-$ rich AGB stars than MacLean et al. 2016). Although the authors do not provide the coordinates of their sample stars, we were able to find 29 RGB stars in common, by investigating the overlap existing between their sample and Marino et al. (2008) and applying our cross-matching criterion with an angular distance $\leq 0.3^{\prime \prime}$. The mean differences in the derived stellar parameters and $\mathrm{Na}$ abundance for this RGB subsample were found to be (in the sense of our result minus theirs) $-142 \pm 78 \mathrm{~K}$ in $T_{\text {eff }},-0.05 \pm 0.13$ in $\log g,-0.04 \pm 0.23 \mathrm{~km} \mathrm{~s}^{-1}$ in $\xi_{\mathrm{t}}$, $-0.01 \pm 0.09$ dex in $[\mathrm{Fe} / \mathrm{H}]$, and $0.04 \pm 0.12 \mathrm{dex}$ in $[\mathrm{Na} / \mathrm{H}]$. Except for the offset in $T_{\text {eff }}$, we consider this to be a very good agreement. Besides, our derived abundances also agree quite well with the results of Marino et al. (2017) who studied 17 AGB stars in this cluster. By comparing 14 common stars, differences of $-0.01 \pm 0.05 \mathrm{dex}$ in $[\mathrm{Fe} / \mathrm{H}]$ and $0.00 \pm 0.07 \mathrm{dex}$ in $[\mathrm{Na} / \mathrm{H}]_{\mathrm{LTE}}$ were found between the two studies (our result minus theirs), while the differences in $T_{\text {eff }}, \log g$, and $\xi_{\text {t }}$ were, respectively, $-69 \pm 54 \mathrm{~K},-0.09 \pm 0.17$, and $-0.22 \pm 0.11 \mathrm{~km} \mathrm{~s}^{-1}$. 

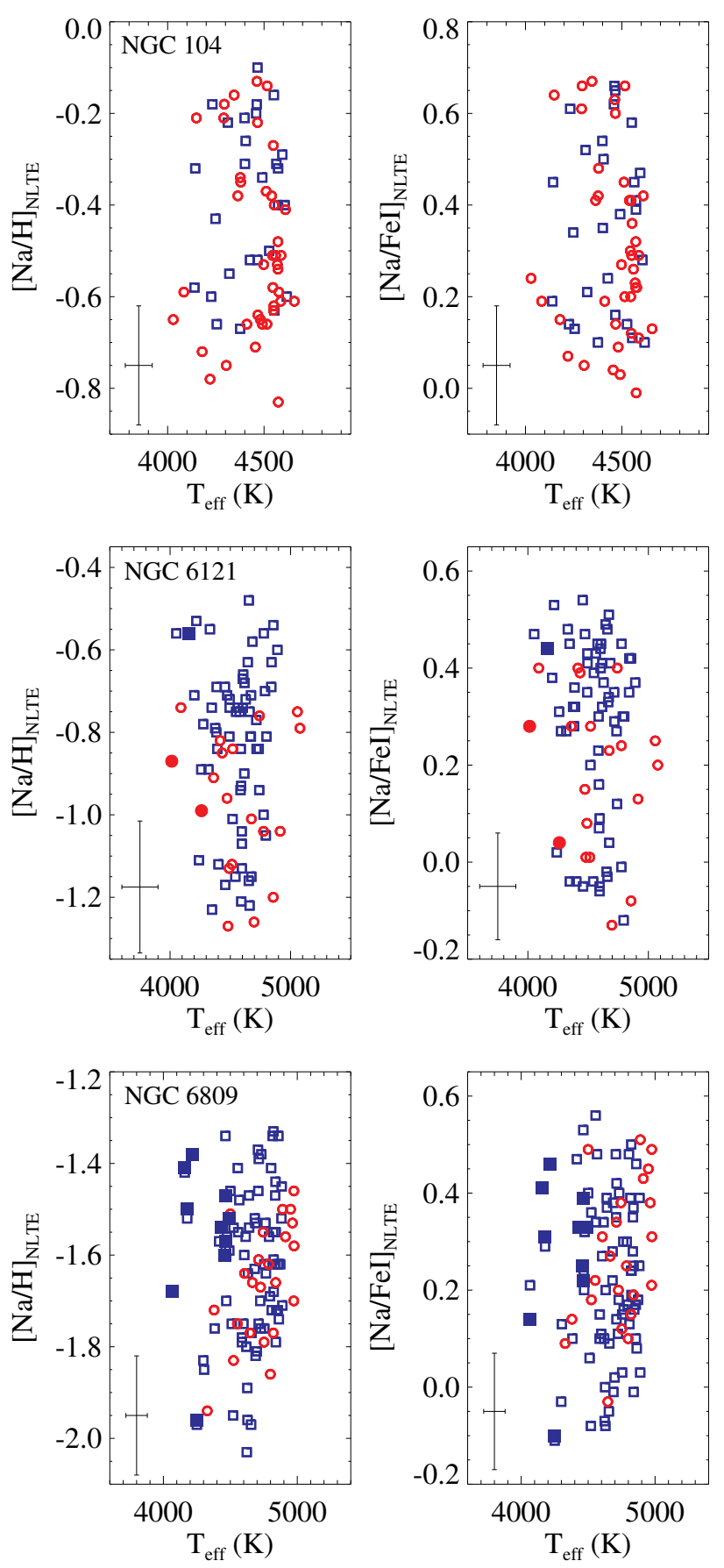

Fig. 2. Abundance distributions of our complete (AGB + RGB) sample in NGC 104, NGC 6121, and NGC 6809 (top, middle, and bottom). Left: $[\mathrm{Na} / \mathrm{H}]_{\mathrm{NLTE}}-T_{\text {eff }}$; right: $[\mathrm{Na} / \mathrm{Fe} \mathrm{I}]_{\mathrm{NLTE}}-T_{\text {eff }}$. Symbols are the same as in Fig. 1. The error bars correspond to our estimates for the GIRAFFE sample.

\subsubsection{NGC 6809}

In NGC 6809 (23 AGB, 77 RGB), we find that the Na abundances of the RGB sample spread more evenly while the AGB stars tend to be more concentrated. The difference between the maximum $[\mathrm{Na} / \mathrm{H}]$ values reached by each sample amounts to 0.12 dex (the AGB sample reaching lower values). The difference is only half of the one observed in NGC 6121, and the two-sided K-S test does not provide any strong evidence for the two distributions to be different, with $D=0.190$ and $p$ value $=0.498$.

This is the first time that AGB stars are targeted and analyzed for their $\mathrm{Na}$ abundances in this cluster. We have however 27 RGB stars in common with the Carretta et al. (2009) sample, for which we found mean differences of $52 \pm 27 \mathrm{~K}$ in $T_{\text {eff }}$, $-0.01 \pm 0.01$ in $\log g,-0.16 \pm 0.38 \mathrm{~km} \mathrm{~s}^{-1}$ in $\xi_{\mathrm{t}}, 0.01 \pm 0.05 \mathrm{dex}$ in $[\mathrm{Fe} \mathrm{I} / \mathrm{H}], 0.10 \pm 0.08 \mathrm{dex}$ in $[\mathrm{Fe} \mathrm{II} / \mathrm{H}]$, and $0.00 \pm 0.19 \mathrm{dex}$ in $[\mathrm{Na} / \mathrm{H}]$. Within the errors, our results are consistent with those derived by Carretta et al. (2009).

\subsubsection{NGC 2808}

For completeness, we recall here the main results of Paper I: the Na abundances of the 33 AGB and 40 RGB stars we analyzed in NGC 2808 can be considered to follow the same distribution (K-S test gives $D=0.268$ and $p$-value $=0.137$ ) although a difference of 0.21 dex was found between the maximum $[\mathrm{Na} / \mathrm{H}]$ values of the AGB and RGB samples, with the AGB maximum value being lower.

\section{Other clusters}

\subsection{Re-analysis of C13 data for NGC 6752}

To enlarge the number of clusters studied self-consistently, we decided to reanalyze the publicly available data of C13 following our analytical methods. We adopted the reddening $E(B-V)=0.04 \mathrm{mag}$ and visual distance modulus $(m-$ $M)_{V}=13.24$ mag from Gratton et al. (2003), and assumed stellar masses of $m_{\mathrm{AGB}}=0.61 M_{\odot}$ and $m_{\mathrm{RGB}}=0.83 M_{\odot}$, following the theoretical predictions of stellar evolution models (assuming an age of $12.5 \mathrm{Gyr}$, Chantereau et al. 2015). Using the methods described in Sects. 3.1 and 3.2, we derived the stellar parameters and the metallicities for a total of 44 individual stars (20 AGB and 24 RGB stars). Overall, we find a good agreement with the $\mathrm{C} 13$ result on the effective temperature with a mean difference of $\Delta T_{\text {eff }}=-24 \pm 51 \mathrm{~K}$ (ours minus $\mathrm{C} 13$ ), while the mean differences on the gravity and microturbulent velocity are $\Delta \log g=$ $-0.14 \pm 0.07$ and $\Delta \xi_{\mathrm{t}}=-0.30 \pm 0.25 \mathrm{~km} \mathrm{~s}^{-1}$, respectively. We derive the following LTE Fe abundances: $[\mathrm{Fe} \mathrm{I} / \mathrm{H}]_{\mathrm{AGB}}=-1.85 \pm$ $0.02 \operatorname{dex}(\sigma=0.06 \mathrm{dex}),[\mathrm{Fe} \mathrm{II} / \mathrm{H}]_{\mathrm{AGB}}=-1.65 \pm 0.06 \operatorname{dex}(\sigma=$ $0.04 \mathrm{dex}),[\mathrm{Fe} \mathrm{I} / \mathrm{H}]_{\mathrm{RGB}}=-1.66 \pm 0.02 \mathrm{dex}(\sigma=0.07 \mathrm{dex})$, and $[\mathrm{Fe} \mathrm{II} / \mathrm{H}]_{\mathrm{RGB}}=-1.55 \pm 0.06 \mathrm{dex}(\sigma=0.05 \mathrm{dex})$, and find for the AGB stars a very good agreement with Lapenna et al. (2016), who re-observed the 20 AGB stars of $\mathrm{C} 13$ at higher resolution with ESO-VLT/UVES $\left([\mathrm{Fe} \mathrm{I} / \mathrm{H}]_{\mathrm{AGB}}=-1.80 \pm 0.01 \mathrm{dex}\right.$ and $\left.[\mathrm{Fe} I \mathrm{II} / \mathrm{H}]_{\mathrm{AGB}}=-1.58 \pm 0.01 \mathrm{dex}\right)$. After applying NLTE corrections to the Fe I abundances (Bergemann et al. 2012; Lind et al. 2012 ), we derived the average values of $[\mathrm{Fe} \mathrm{I} / \mathrm{H}]_{\mathrm{NLTE}, \mathrm{AGB}}=$ $-1.76 \pm 0.04 \mathrm{dex}$ and $[\mathrm{Fe} \mathrm{I} / \mathrm{H}]_{\mathrm{NLTE}, \mathrm{RGB}}=-1.60 \pm 0.04 \mathrm{dex}$, which bring the Fe I-Fe II values for the RGB stars into agreement (within the errors) and almost halves the Fe I-Fe II difference for the AGB stars. Compared to the value assumed by C13 for all their sample stars $([\mathrm{Fe} / \mathrm{H}]=-1.54 \mathrm{dex})$, our derived metallicities are slightly lower and point to a 0.1 dex difference between AGB and RGB stars.

Considering the weakness of the $6154-60 \AA \mathrm{Na}$ lines in a non-negligible number of spectra of the $\mathrm{C} 13$ sample, we followed their choice and derived the $\mathrm{Na}$ abundances from the EWs of the Na doublet at 5682-5688 $\AA^{3}$. We apply the same NLTE

3 The choice of the Na doublet has only a negligible impact on the derived abundances $(-0.027 \mathrm{dex}, \sigma=0.073 \mathrm{dex}$, bluer - redder doublet). 
Y. Wang et al.: Sodium abundances of AGB and RGB stars in Galactic globular clusters. II.
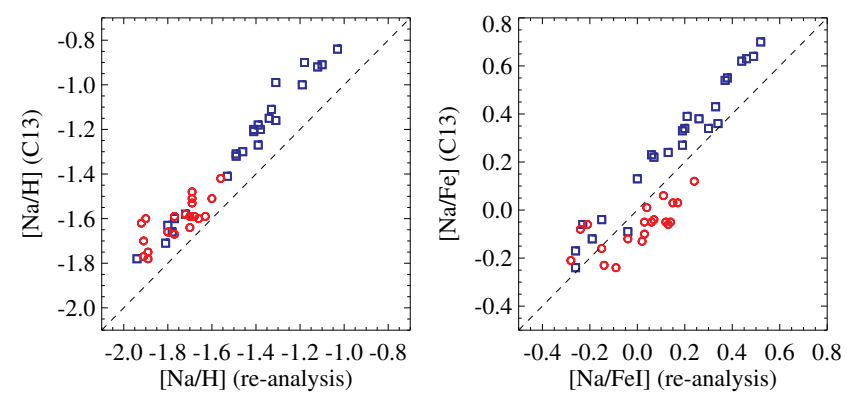

Fig. 3. Comparison of the Na abundance (NLTE) of NGC 6752 sample between our derived results and those of $\mathrm{C} 13$. Red circles represent AGB stars, and blue squares represent RGB stars.

corrections (Lind et al. 2011) to the derived $\mathrm{Na}$ abundances as for the other GCs and find a mean difference (ours minus C13) of $-0.16 \pm 0.06 \mathrm{dex}$ in $[\mathrm{Na} / \mathrm{H}]$ and of $-0.03 \pm 0.13 \mathrm{dex}$ in $[\mathrm{Na} / \mathrm{Fe}]$. We compare the derived $\mathrm{Na}$ abundances from the two studies in Fig. 3. The differences in $[\mathrm{Na} / \mathrm{H}]$ appear to be systematic (likely due to the combination of different stellar parameters and adopted solar abundances), while the effect on $[\mathrm{Na} / \mathrm{Fe}]$ is influenced mainly by the adopted $\mathrm{Fe}$ abundance (overall cluster metallicity in the case of $\mathrm{C} 13$, individual stellar metallicities in our case; see also Lapenna et al. 2016). The remaining offsets are likely to come from the NLTE correction applied to the $\mathrm{Na}$ abundance (C13 used the Gratton et al. 1999, values).

Figure 4 shows all results of this re-analysis (left column), with a direct comparison to the values published by $\mathrm{C} 13$ (middle column). For AGB stars, our $[\mathrm{Na} / \mathrm{H}]$ distribution agrees quite well with that of $\mathrm{C} 13$, while this is not the case for $[\mathrm{Na} / \mathrm{Fe}]$. Testing the assumption on the metallicities made by the two analyses shows that we can fully reproduce $\mathrm{C} 13$ results as soon as we use an overall metallicity value for the cluster. Both our $[\mathrm{Na} / \mathrm{H}]$ and $[\mathrm{Na} / \mathrm{Fe} \mathrm{I}]$ distributions derived for the AGB sample agree well with those derived by Lapenna et al. (2016) from higherresolution spectral data, as shown in the right column of Fig. 4 (labeled as L16; the RGB stars are from our re-analysis and are shown only to aid the comparison), which supports our abundance results. As in Sect. 3.6, we take $[\mathrm{Na} / \mathrm{H}]$ as the $\mathrm{Na}$ abundance indicator and find that there is a significant lack of Na-rich AGB stars in the sample of NGC 6752 (2P AGB stars account for $\sim 15 \%$, cf. Sect. 5). However, Lapenna et al. (2016) claimed that both $1 \mathrm{P}$ and $2 \mathrm{P}$ stars populate the AGB of NGC 6752 with $~ 65 \%$ of AGB stars belonging to $2 \mathrm{P}$ based on the $[\mathrm{O} / \mathrm{Fe}]-[\mathrm{Na} / \mathrm{Fe}]$ distribution. Only stars with extreme $\mathrm{Na}$ enhancements are claimed to be missing in their AGB sample. We note, however, that this conclusion was derived based on $[\mathrm{Na} / \mathrm{Fe}]$ ratios. The Fe I-Fe II discrepancy (especially their different behaviors in AGB and RGB stars) may affect the relative $\mathrm{Na}$ abundance distributions between AGB and RGB stars (e.g., Fig. 4, left column) so that the $[\mathrm{Na} / \mathrm{Fe}]$ indicator carries a larger uncertainty compared to the $[\mathrm{Na} / \mathrm{H}]$ (Sect. 3.6). Furthermore, we note that our derived fraction of $2 \mathrm{P}$-AGB stars $(\sim 15 \%)$ is lower than the one predicted by Cassisi et al. $(2014, \sim 50 \%)$ based on simulations of the horizontal branch. While the specific fraction numbers of different stellar populations depend on the adopted separation criteria (see also Sect. 5.3), the sampling could also affect the results. Although we believe that this GC deserves further scrutiny, for the sake of a coherent discussion, we use our derived abundance ratios in the following sections.
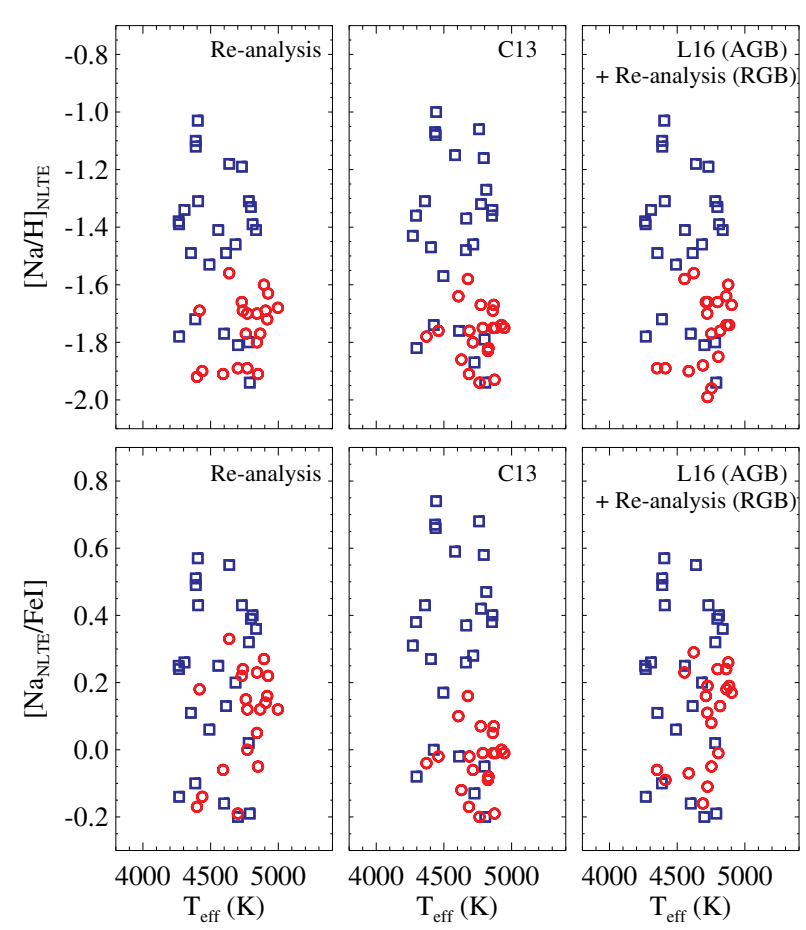

Fig. 4. Na abundance (NLTE) distributions of the sample of C13 in NGC 6752. The left-column panels show our re-analysis results, middlecolumn panels show the $\mathrm{Na}$ abundance distribution from $\mathrm{C} 13$, and the right-column panels show the Na abundance of the AGB sample derived by Lapenna et al. (2016) together with the RGB stars from our re-analysis. For convenience of comparison, the data from $\mathrm{C} 13$ and Lapenna et al. (2016) are shifted systematically to our scale with a constant which is equal to the mean difference between their results and ours, while the dispersions derived in each study are kept. Red circles and blue squares represent AGB and RGB stars, respectively.

\subsection{Literature data for four additional GCs}

In the literature, one finds four other GCs whose AGB stars have been targeted for their $\mathrm{Na}$ abundances through moderate- and high-resolution spectroscopic observations: NGC 5904 (Ivans et al. 2001; Lai et al. 2011), NGC 5986 (Johnson et al. 2017), NGC 6205 (Johnson \& Pilachowski 2012), and NGC 6266 (Lapenna et al. 2015). The Na abundance patterns observed in these clusters are shown in Fig. 6, where the data points have already been adjusted to be on the same solar abundance and NLTE-correction scales as our own data set; for NGC 5904 the abundance data from Lai et al. (2011) have been unified to the system of Ivans et al. (2001) according to the common star between the two studies, while for the common star we adopt the data derived by Ivans et al.

NGC $6205(\mathrm{M} \mathrm{13} ;[\mathrm{Fe} / \mathrm{H}]=-1.57$, Johnson \& Pilachowski 2012) seems to be relatively devoid of Na-normal AGB stars, with a difference of $\sim 0.4$ dex between the $[\mathrm{Na} / \mathrm{H}]$ minimum values in the RGB and the AGB samples. But, as suggested by García-Hernández et al. (2015), due to the difficulty of distinguishing AGB from RGB stars at the bright end of the giant branch, some misclassification might have occurred, which could slightly favor the RGB sample. Notwithstanding, the presence of a large fraction of Na-rich AGB stars in NGC 6205 is clear.

NGC $5986([\mathrm{Fe} / \mathrm{H}]=-1.54$, Johnson et al. 2017) shows comparable $[\mathrm{Na} / \mathrm{H}]$ spreads in AGB and RGB star samples with the maximum $[\mathrm{Na} / \mathrm{H}]$ value of AGB stars being 0.14 dex lower 
Table 10. Na abundance spread, critical value of the Na abundance distinguishing $1 \mathrm{P}$ and $2 \mathrm{P}$ stars, and the corresponding fraction of Na-rich $2 \mathrm{P}$ stars $f_{2 \mathrm{P}}$ (together with the associated errors based on Beta distribution) in the RGB and AGB samples.

\begin{tabular}{cccccc|cccc}
\hline \hline & NGC 6809 & NGC 6121 & NGC 2808 & NGC 104 & NGC 6752 & NGC 6205 & \multicolumn{2}{c}{ NGC 5986 } & \multicolumn{2}{c}{ NGC 5904 } & NGC 6266 \\
& M 55 & M 4 & & 47 Tuc & & M 13 & & M 5 & M 62 \\
\hline$[\mathrm{Na} / \mathrm{H}]_{\text {cri }}$ & -1.70 & -0.97 & -1.05 & -0.50 & -1.65 & -1.50 & -1.46 & -1.35 & -1.05 \\
\hline$\Delta[\mathrm{Na} / \mathrm{H}]_{\mathrm{AGB}}$ & 0.48 & 0.53 & 0.54 & 0.70 & 0.36 & 0.62 & 0.61 & 0.51 & 0.30 \\
$f_{2 \mathrm{P}, \mathrm{AGB}}$ & $65 \pm 11$ & $53 \pm 11$ & $55 \pm 9$ & $40 \pm 8$ & $15 \pm 11$ & $87 \pm 13$ & $57 \pm 18$ & $67 \pm 13$ & $0 \pm 23$ \\
\hline$\Delta[\mathrm{Na} / \mathrm{H}]_{\mathrm{RGB}}$ & 0.70 & 0.75 & 0.75 & 0.57 & 0.91 & 1.12 & 0.76 & 0.68 & 1.08 \\
$f_{2 \mathrm{P}, \mathrm{RGB}}$ & $66 \pm 6$ & $76 \pm 6$ & $52 \pm 8$ & $67 \pm 10$ & $75 \pm 11$ & $76 \pm 5$ & $72 \pm 12$ & $69 \pm 9$ & $62 \pm 14$ \\
\hline$[\mathrm{Fe} / \mathrm{H}]$ & -1.86 & -1.14 & -1.11 & -0.82 & -1.60 & -1.57 & -1.54 & -1.22 & -1.05 \\
Age & 13.00 & 11.50 & 11.00 & 11.75 & 12.50 & 12.00 & 12.25 & 11.50 & 11.60 \\
& \pm 0.25 & \pm 0.38 & \pm 0.38 & \pm 0.25 & \pm 0.25 & \pm 0.38 & \pm 0.75 & \pm 0.25 & \pm 0.60 \\
$M_{\mathrm{V}}$ & -7.57 & -7.19 & -9.39 & -9.42 & -7.73 & -8.55 & -8.44 & -8.81 & -9.18 \\
mass & 0.269 & 0.195 & 1.420 & 1.500 & 0.317 & 0.775 & 0.599 & 0.857 & 1.220 \\
$\mathrm{HBR}$ & 0.87 & -0.06 & -0.49 & -0.99 & 1.00 & 0.97 & 0.97 & 0.31 & 0.32 \\
$r_{\mathrm{h}}$ & 4.46 & 2.34 & 2.12 & 3.65 & 2.72 & 3.34 & 3.18 & 4.60 & 2.47 \\
$r_{\mathrm{t}}$ & 25.10 & 20.79 & 43.42 & 56.10 & 64.39 & 56.40 & 31.83 & 61.96 & 18.00 \\
ellipticity & 0.02 & 0.00 & 0.12 & 0.09 & 0.04 & 0.11 & 0.06 & 0.14 & 0.01 \\
c.c. & 0.93 & 1.65 & 1.56 & 2.07 & 2.50 & 1.53 & 1.23 & 1.73 & 1.71 \\
$\sigma_{\mathrm{v}}$ & 4.0 & 4.0 & 13.4 & 11.0 & 4.9 & 7.1 & - & 5.5 & 14.3 \\
\hline
\end{tabular}

Notes. The five GCs on the left side of the vertical line are the GCs analyzed (or re-analyzed) by ourselves homogeneously, and their [Fe/H] are the values derived by us, while the four GCs on the right are collected from the literature (see the text). The other global GC properties are from the literature. The GC ages (Gyr) are adopted from VandenBerg et al. (2013), except NGC 6266 whose age is from Roediger et al. (2014); the $M_{\mathrm{V}}$ (mag), ellipticities, c.c., and $\sigma_{\mathrm{v}}\left(\mathrm{km} \mathrm{s}^{-1}\right)$ are from Harris $\left(1996,2010\right.$ version); the masses $\left(\times 10^{6} M_{\odot}\right)$ are adopted from Boyles et al. (2011); the $\mathrm{HBR}, r_{\mathrm{h}}(\mathrm{pc})$, and $r_{\mathrm{t}}(\mathrm{pc})$ are from Mackey \& van den Bergh (2005).

than that of RGB stars. The sample of AGB stars is, however, rather limited.

NGC 5904 (M5; $[\mathrm{Fe} / \mathrm{H}]=-1.22$, Ivans et al. 2001; Lai et al. 2011) shows a paucity of very Na-rich AGB stars compared to RGB stars, with a difference of 0.25 dex between the maximum $[\mathrm{Na} / \mathrm{H}]$ value of the AGB and RGB samples.

NGC 6266 (M62; $[\mathrm{Fe} / \mathrm{H}]=-1.05$, Lapenna et al. 2015) makes itself distinct by showing no Na-rich AGB star in the small sample analyzed by Lapenna et al. (2015). However, in our opinion, no firm conclusion can be drawn because the AGB phase may not be sufficiently sampled.

\section{Discussion for the full sample of GCs with $\mathrm{Na}$ abundance determination on the AGB}

\subsection{Comparison criteria}

For the reasons previously described, we use the $[\mathrm{Na} / \mathrm{H}]_{\mathrm{NLTE}}$ data to discuss the differences and similarities in terms of $\mathrm{Na}$ abundances in the nine GCs for which both the RGB and AGB have been studied. The abundance determination is self-consistent for five GCs (NGC 6809, NGC 6121, NGC 2808, NGC 104, and NGC 6752; this paper and Paper I). For the four other GCs (NGC 6205, NGC 5986, NGC 5904, and NGC 6266), we use the data from the original papers that we modified to have consistent Na reference solar abundance and NLTE corrections (Sect. 4.2).

We use the same definition as in Paper I to distinguish Nanormal and Na-rich stars (often called $1 \mathrm{P}$ and $2 \mathrm{P}$ stars in the literature; see e.g., Prantzos \& Charbonnel 2006; Carretta et al. 2009), the latter ones being defined as those having $[\mathrm{Na} / \mathrm{H}]$ higher than $[\mathrm{Na} / \mathrm{H}]_{\text {cri }}=[\mathrm{Na} / \mathrm{H}]_{\min }+0.3$ dex, where $[\mathrm{Na} / \mathrm{H}]_{\min }$ is the minimum $\mathrm{Na}$ value derived for the $\mathrm{RGB}+\mathrm{AGB}$ sample in a given cluster and 0.3 dex is about one third of the $[\mathrm{Na} / \mathrm{H}]$ spread. Table 10 gathers the values of $[\mathrm{Na} / \mathrm{H}]_{\mathrm{cri}}$, of the fraction of $\mathrm{Na}-$ rich $\mathrm{RGB}$ and $\mathrm{AGB} 2 \mathrm{P}$ stars $\left(f_{2 \mathrm{P}, \mathrm{RGB}}\right.$ and $f_{2 \mathrm{P}, \mathrm{AGB}}$ respectively), and of the Na spreads $\Delta[\mathrm{Na} / \mathrm{H}]$ for the RGB and AGB subsamples in each GC; we have also collected in the table important cluster characteristics that are relevant for the discussion.

\subsection{Na abundance distributions among the RGB and $A G B$ samples}

We gather all the $[\mathrm{Na} / \mathrm{H}]_{\mathrm{NLTE}}$ data for the nine GCs as a function of stellar effective temperature (Figs. 5 and 6) and in the form of continuous histograms (Figs. 7 and 8, where every star is represented by a Gaussian profile with a weight of one and standard deviation equalling the uncertainty on the measurement) for RGB and AGB samples. In all cases, the Na dispersion observed for RGB and AGB stars does not depend on the effective temperature (Figs. 5 and 6) or the brightness of the stars (not shown here). This means that there is no in situ evolution effect that modifies the $\mathrm{Na}$ abundance inside the GC evolved stars we observe today.

A quick look at these figures shows that in the majority of the GCs under scrutiny (eight out of nine, the only exception being NGC 104), the Na spread is smaller among AGB stars than among RGB stars (see the actual dispersion numbers in Table 10). More specifically, the maximum $[\mathrm{Na} / \mathrm{H}]$ values derived for the AGB stars are lower than the ones derived for the RGB stars. In three out of these eight GCs (NGC 6809, NGC 6205, and NGC 5986), the maximum values for $\mathrm{Na}$ on the RGB and the AGB are however marginally consistent considering the errors. Three clusters (NGC 2808, NGC 5904, NGC 6121) clearly lack the most Na-rich AGB star. Finally, the last two clusters (NGC 6752 and NGC $6266^{4}$ ) stand out by showing (almost) no Na-rich AGB stars (i.e., with $[\mathrm{Na} / \mathrm{H}]$ higher than $\left.[\mathrm{Na} / \mathrm{H}]_{\mathrm{cri}}\right)$ in the samples analyzed so far.

\footnotetext{
4 Larger uncertanity may exist for this cluster considering the paucity of stars that have been studied so far (Lapenna et al. 2015).
} 
Y. Wang et al.: Sodium abundances of AGB and RGB stars in Galactic globular clusters. II.
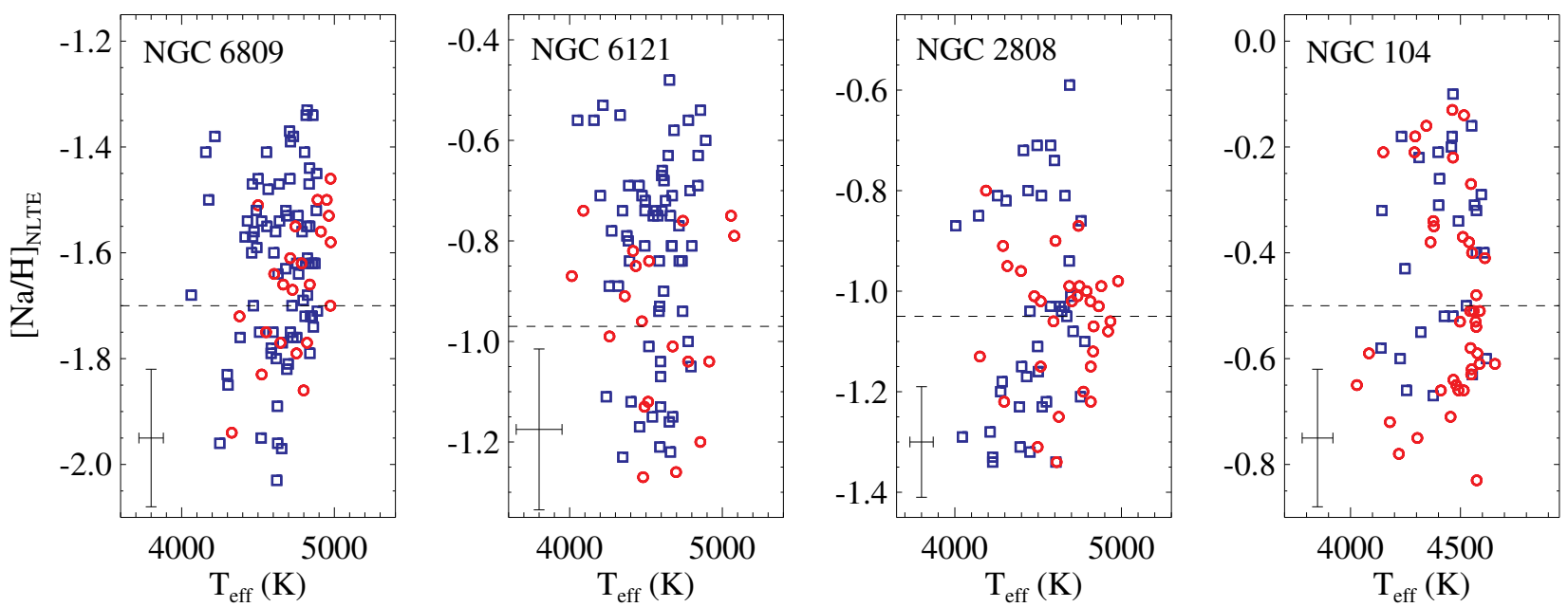

Fig. 5. $[\mathrm{Na} / \mathrm{H}]$ versus $T_{\text {eff }}$ of the AGB and RGB stars (red and blue symbols, respectively) in NGC 6809, NGC 6121, NGC 2808, and NGC 104 from the present analysis and Paper I. The horizontal black dashed lines mark the critical $[\mathrm{Na} / \mathrm{H}]$ ratio separating roughly the Na-poor $1 \mathrm{P}$ and Na-rich 2P stars according to Carretta et al. (2009) criteria. The clusters are presented by increasing [Fe/H] from left to right.
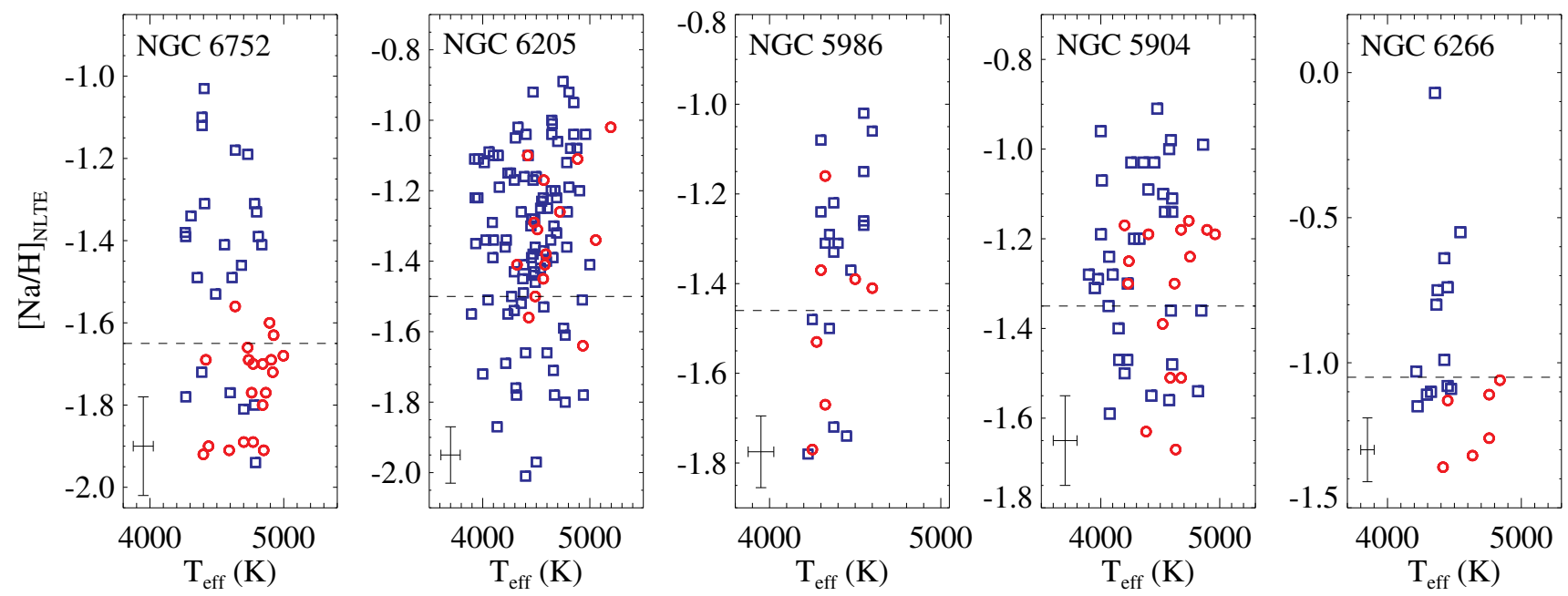

Fig. 6. Same as Fig. 5 but for NGC 6752, NGC 6205, NGC 5986, NGC 5904, and NGC 6266. The values for NGC 6752 are from our re-analysis of C13 data, the others are from the literature (see the text) but the adopted solar abundance and the NLTE correction for Na have been homogenized to those we used for our own GCs.

The continuous histograms of the $[\mathrm{Na} / \mathrm{H}]$ (Figs. 7 and 8 ) indicate that the $\mathrm{Na}$ abundance distributions of the RGB and AGB stars cannot be described by one type of profile (single- or double-peak) and confirm that they vary from cluster to cluster. According to the current data, both AGB and RGB samples of NGC 104 and NGC 6205 are bimodal; those of NGC 6121 and NGC 6809 are unimodal; for NGC 2808 and NGC 6752, their RGB and AGB samples appear to be bimodal and unimodal, respectively; NGC 6266 has a unimodal AGB sample and trimodal RGB sample; NGC 5904 shows bimodal distribution in AGB sample but a broadened profile in RGB sample which indicates that three closely located peaks may exist; while NGC 5986 shows trimodal distributions in both AGB and RGB samples. Separations can clearly be found between the main peaks of AGB and RGB samples of NGC 6121, NGC 6752, NGC 6266, and NGC 5986, with the ones of AGB always having lower $[\mathrm{Na} / \mathrm{H}]$ values than RGB, and the main peaks in NGC 6752 separate the most.

\subsection{Fractions of $1 P$ and $2 P$ stars}

The number ratio between $1 \mathrm{P}$ and $2 \mathrm{P}$ stars has been extensively used to constrain the models that aim at explaining the chemical properties of the stellar populations in GCs (e.g., Prantzos \& Charbonnel 2006; Carretta et al. 2010; Decressin et al. 2010; Schaerer \& Charbonnel 2011; Charbonnel et al. 2014; Larsen et al. 2014; Bastian \& Lardo 2015; Khalaj \& Baumgardt 2015). As a general concept, 1P and 2P refer respectively to GC stars that present chemical abundances similar or different to those of field stars of similar metallicity. This can be seen in Fig. 9 where we compare the Na data for RGB and AGB stars that we use in the discussion (including our determinations and data from the literature) with the $\mathrm{Na}$ data in Galactic field stars gathered and homogenized by Carretta (2013). Using the same definition of $[\mathrm{Na} / \mathrm{H}]_{\text {cri }}$ as in Paper I (see Sect. 5.1), we calculate the fraction of $2 \mathrm{P}$ stars $\left(f_{2 \mathrm{P}}\right)$ both on the RGB and AGB in each individual GC (Table 10). 

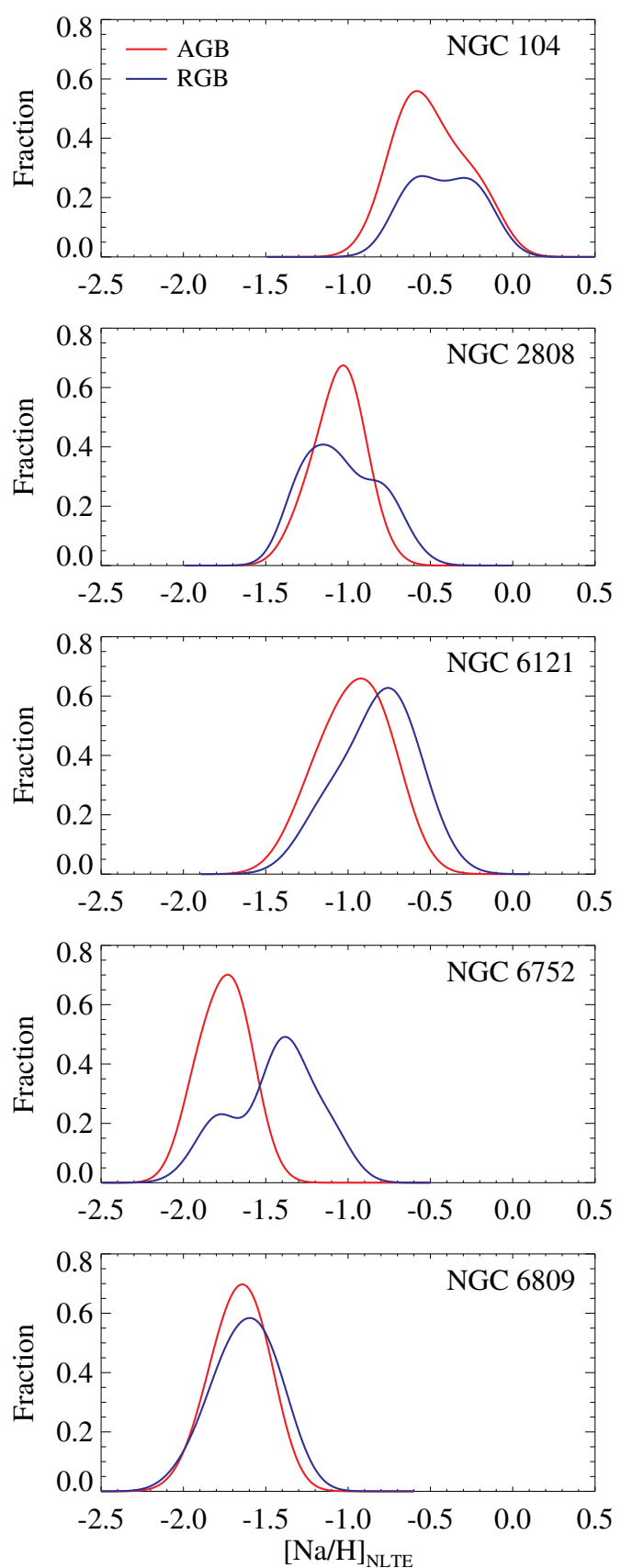

Fig. 7. Continuous histograms of $[\mathrm{Na} / \mathrm{H}]$ of the $\mathrm{AGB}$ (red) and RGB (blue) samples for the five GCs we have analyzed in a self-consistent way (whose $[\mathrm{Fe} / \mathrm{H}]$ decrease from top to bottom).

Our results agree with the finding of Carretta et al. (2009) that the 2P RGB component is present in all clusters, with a fraction between $\sim 50 \%$ and $75 \%$. For the AGB, we confirm previous abundance studies that revealed a complex picture, with some clusters being almost devoid of 2P AGB stars (NGC 6752, NGC 6266), some having similar 2P fractions on the AGB to on the RGB (NGC 2808, NGC 5904, and NGC 6809), and all the possible intermediate cases between these two extreme behaviors. Overall, the fraction of $2 \mathrm{P}$ AGB stars varies between $\sim 0 \%$ and $87 \%$ (these extreme values corresponding to NGC 6266 and NGC 6205, respectively).

Obviously, the actual numbers one obtains for the fractions of $1 \mathrm{P}$ and $2 \mathrm{P}$ stars in a given GC depend on the adopted
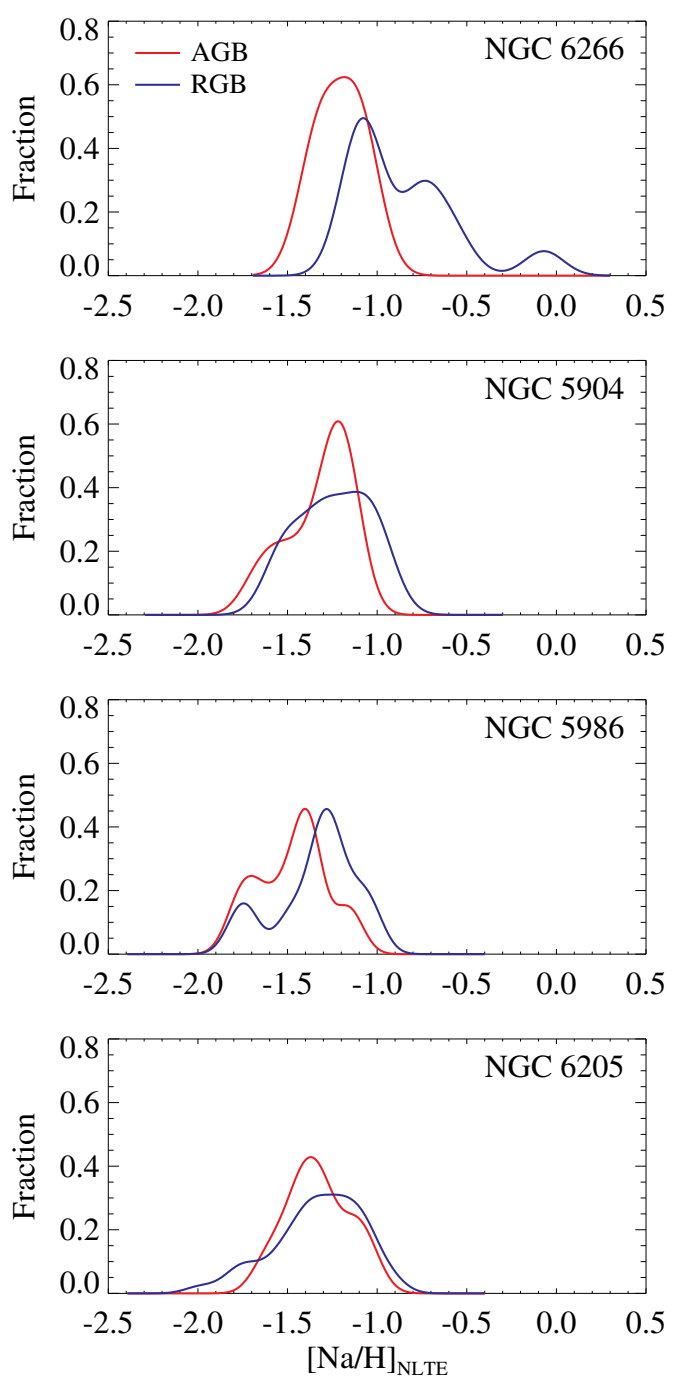

Fig. 8. Same as Fig. 7 but for literature data.

distinction criteria. For example, our conclusion that NGC 6121 hosts 2P AGB stars seems at first sight to contradict the claim by MacLean et al. (2016) that there is no 2P AGB star in this cluster. However, MacLean et al. (2016) derived the population separation point (PSP) for their NGC 6121 sample $([\mathrm{Na} / \mathrm{O}]=-0.16$ dex $)$ by identifying a minimum in the $[\mathrm{Na} / \mathrm{O}]$ distribution between the two subpopulations in the RGB sample of Marino et al. (2008), and they found that the abundance distribution was consistent with all the AGB stars being of $1 \mathrm{P}$, while $45 \%$ of the RGB stars belong to $2 \mathrm{P}$. However, if the criterion by Carretta et al. (2009) to distinguish 1P and 2P stars (i.e., $\left.[\mathrm{Na} / \mathrm{Fe}]_{\text {cri }}=[\mathrm{Na} / \mathrm{Fe}]_{\min }+0.3 \mathrm{dex}\right)$ is applied, one finds 6 out of 15 AGB stars and 65 out of 106 RGB stars belonging to 2P, which accounts for $40 \%$ and $61 \%$ of their AGB and RGB sample, respectively. Although it is still slightly different from the fractions found for our own sample (53\% AGB and 76\% RGB stars belonging to $2 \mathrm{P}$ ), it is possible to identify some $2 \mathrm{P}$ AGB stars from the $\mathrm{Na}$ abundance distributions of both samples (ours and MacLean et al. 2016) when the same 1P-2P separation criterion is adopted. Moreover, from the $[\mathrm{O} / \mathrm{Fe}]-[\mathrm{Na} / \mathrm{Fe}]$ distribution of the AGB stars studied by Marino et al. (2017) we can infer that the Na-rich/O-poor stars account for $47 \%$, which is close to our $2 \mathrm{P}$ AGB fraction of $53 \%$. The photometric studies 

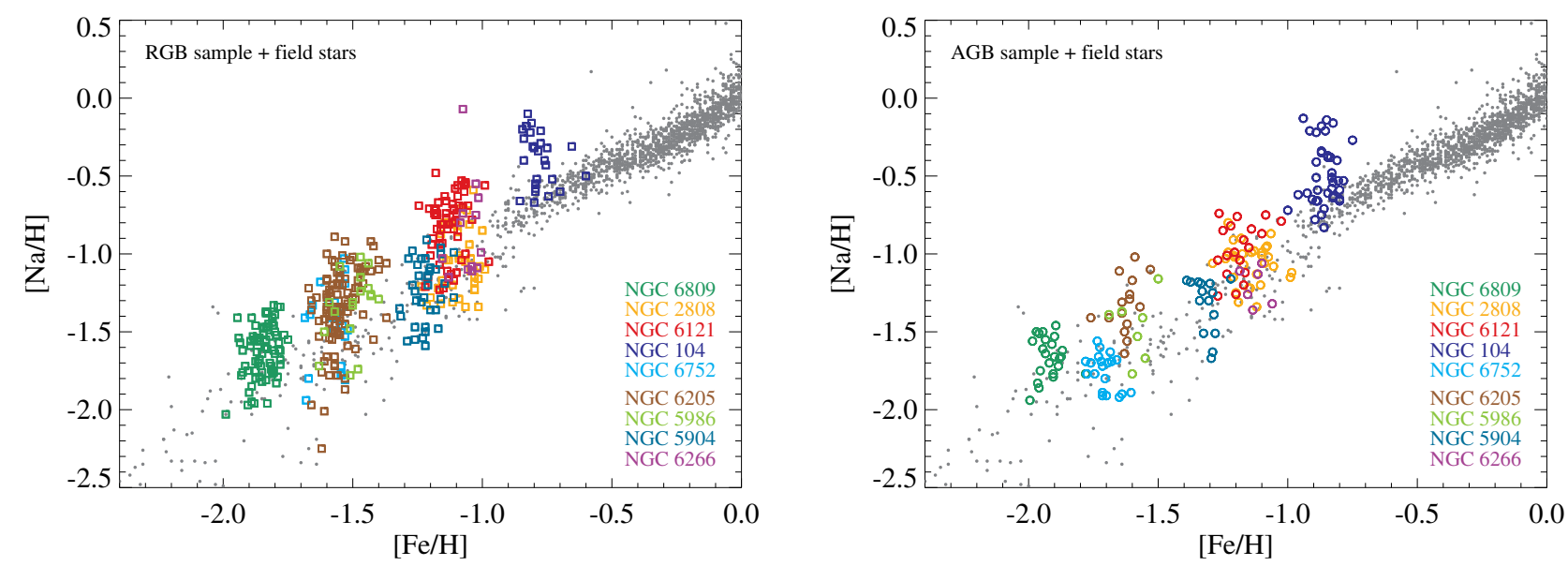

Fig. 9. $[\mathrm{Na} / \mathrm{H}]$ (NLTE) as a function of $[\mathrm{Fe} / \mathrm{H}]$ for RGB (left) and AGB (right) stars in the nine GCs discussed in this paper (our analysis for NGC 104, NGC 2808, NGC 6121, NGC 6809, and NGC 6752; literature data for NGC 5904, NGC 5986, NGC 6205, and NGC 6266) and in Galactic field stars from Carretta (2013, gray points).

of Marino et al. (2017) and Lardo et al. (2017) also support the claim that NGC 6121 hosts multiple populations on AGB. Thus we believe that this cluster should have more than one stellar population along its AGB.

\subsection{Dependencies of the AGB population fraction and theoretical considerations}

\subsubsection{Dependencies of the AGB population fraction on the GC parameters}

We investigate possible dependencies between the fraction of 2P AGB stars $\left(f_{2 \mathrm{P}, \mathrm{AGB}}\right)$ we derived and the GC global parameters listed in Table 10 . We consider $[\mathrm{Fe} / \mathrm{H}]$, age, absolute $V$ magnitude $\left(M_{V}\right)$, mass, HB morphology index (HBR), half-light radius $\left(r_{\mathrm{h}}\right)$, tidal radius $\left(r_{\mathrm{t}}\right)$, ellipticity, central concentration (c.c.), and central velocity dispersion $\left(\sigma_{\mathrm{v}}\right)$. Figure 10 shows the data points and the linear fits derived by least square fitting with the errors taken into account. The Pearson correlation coefficients for each set of data points are also listed as a reference. When we consider only the five GCs that we have analyzed in a consistent manner (black points and solid lines), we obtain weak positive correlations between $f_{2 \mathrm{P}, \mathrm{AGB}}$ and half-light radius; and anticorrelations with GC age (weak), tidal radius, and central concentration. When taking all nine clusters together into account (dashed lines), weak positive relations exist between $f_{2 \mathrm{P}, \mathrm{AGB}}$ and halflight radius and ellipticity; and negative relations are shown between $f_{2 \mathrm{P}, \mathrm{AGB}}$ and $[\mathrm{Fe} / \mathrm{H}]$ (weak), central concentration, and central velocity dispersion (weak). If the two extreme GCs showing almost no 2P AGB stars (NGC 6752 and NGC 6266) are disregarded (dash-dotted lines), $f_{2 \mathrm{P}, \mathrm{AGB}}$ correlates positively with $\mathrm{GC}$ age, HBR, and half-light radius (weak); while the dependencies on $[\mathrm{Fe} / \mathrm{H}], M_{\mathrm{V}}$, mass, central concentration, and central velocity dispersion are negative. Overall, only the anticorrelation between $f_{2 \mathrm{P}, \mathrm{AGB}}$ and central concentration agrees among the fits of all three subsamples. Except for central concentration, the inconsistent results of the three sets of fits indicate that there is no robust dependency for $f_{2 \mathrm{P}, \mathrm{AGB}}$ on any other global GC properties.

\subsubsection{Theoretical considerations}

Charbonnel et al. (2013) proposed that the lack of sodium-rich AGB stars in NGC 6752 could be due to a correlation between
$\mathrm{Na}$ and $\mathrm{He}$ enrichment in the initial mixture of the low-mass stars we observe today, as predicted in the original FRMS scenario (Decressin et al. 2007b). They showed that in this framework, 2P stars born with an initial He abundance above a cutoff value (and consequently with an initial $\mathrm{Na}$ abundance above a cutoff value) are predicted to miss the AGB and evolve directly towards the white dwarf stage after central He burning, because of the impact of helium on stellar evolution. Charbonnel \& Chantereau (2016) showed that when one assumes the same initial $\mathrm{Na}-\mathrm{He}$ correlation for all GCs within the FRMS framework as well as standard mass-loss rates on the RGB, the maximum Na content expected for $2 \mathrm{P}$ stars on the AGB is a function of both the metallicity and the age of GCs. At a given $[\mathrm{Fe} / \mathrm{H}]$, younger clusters are expected to host AGB stars exhibiting a larger $\mathrm{Na}$ spread than older clusters; and, at a given age, higher Na dispersion along the AGB is predicted in metal-poor GCs than in the metal-rich ones. This is depicted in Fig. 11 where we show the model predictions for the ratio between the Na spread on the AGB and that on the RGB as a function of GC age and for different metallicities.

Among the nine currently available GCs, we have two pairs of GCs with similar metallicities, that is, NGC 2808 and NGC 6266, and NGC 2808 and NGC 6121, with the relatively younger $\mathrm{GC}$ in each pair always having larger $[\mathrm{Na} / \mathrm{H}]$ dispersion and 2P-AGB fraction. NGC 5986, NGC 6205, and NGC 6752 have comparable metallicities and slightly different ages, but still the relatively younger $\mathrm{GC}$ has more Na-rich 2P AGB stars. Besides, NGC 5904, NGC 6121, and NGC 6266 have almost the same age but slightly different metallicities, with the most metal-poor one showing the largest number of $2 \mathrm{P}$ AGB stars. In other words, we have five GCs that lie in the domain where the AGB stars are expected to present large $\mathrm{Na}$ abundance dispersions within the FRMS framework. These are NGC 104 and NGC 2808 that are relatively young and metalrich, NGC 6205 and NGC 5986 that have medium age and metallicity, and NGC 6809 which is old and metal-poor. On the other hand, according to the FRMS prediction and based on their metallicities and ages, NGC 5904, NGC 6121, NGC 6752, and NGC 6266 are expected to have their AGB stars showing smaller $\mathrm{Na}$ abundance dispersions compared to their RGB counterparts. Thus, and to the very first order, the observations of these subgroups of GCs are consistent with the trends with age and metallicity predicted by the FRMS scenario.

However, there are several important issues that might blur the general trends one tries to identify (see also the discussion in 

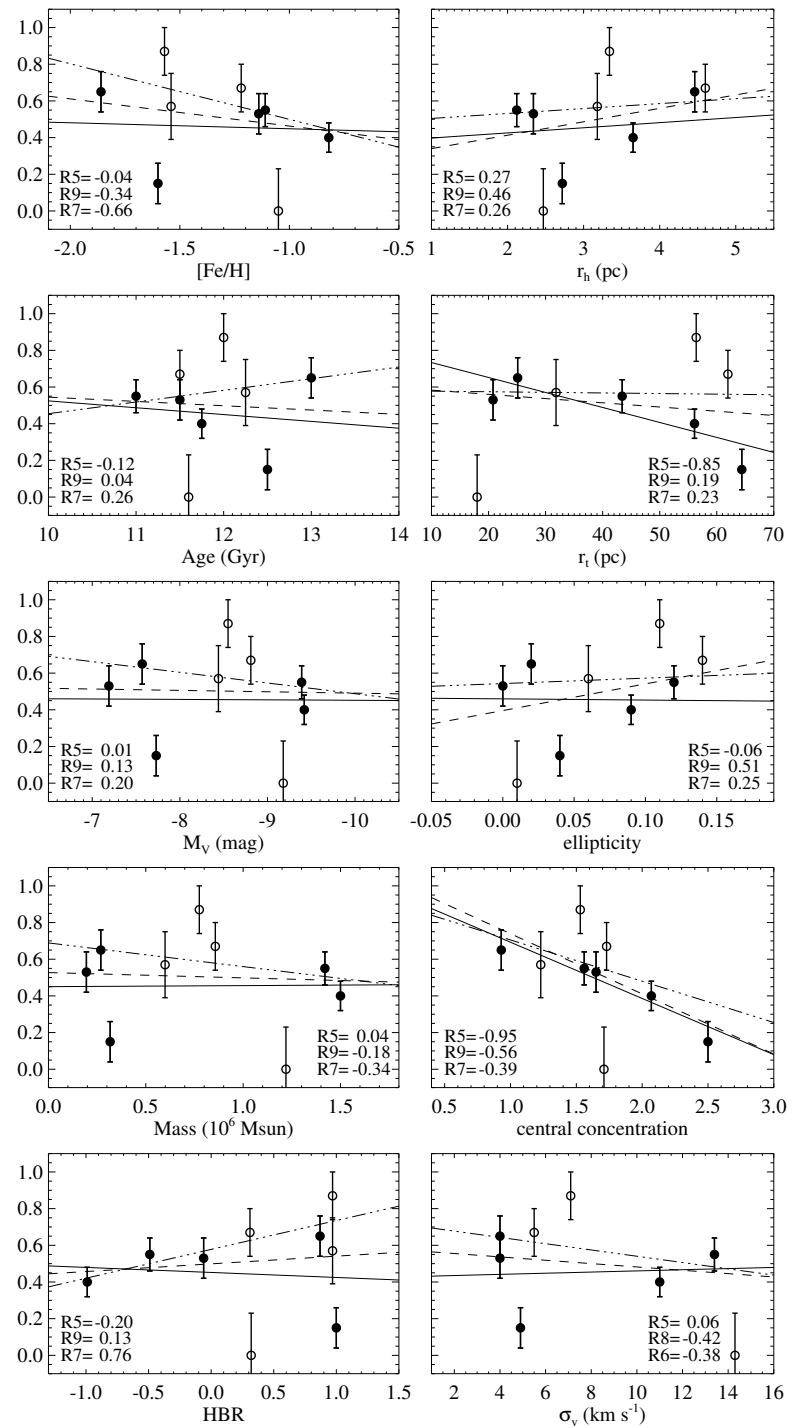

Fig. 10. Relations between the fraction of $2 \mathrm{P}$ AGB stars $\left(f_{2 \mathrm{P} . \mathrm{AGB}}\right)$ and the selected GC global parameters. The data points were all derived using the criteria defined in Sect. 5.1. Filled and open circles are used, respectively, for the five GCs we analyzed self-consistently (NGC 6809, NGC 2808, NGC 6121, NGC 104, and NGC 6752) and for those we took from the literature (NGC 6205, NGC 5986, NGC 5904, and NGC 6266). The associated error bars are computed from Beta distribution. The lines are the linear fits derived by least square fitting (with the errors taken into account); the solid and dashed lines are fitted from our own five GCs and all the nine GCs, respectively, while the dashdotted ones represent the fits disregarding the two most scattered points of NGC 6266 and NGC 6752. The Pearson correlation coefficients considering the five (R5), nine (R9), and seven (R7) data points, respectively, are listed at the bottom of each panel (except for the very bottom right panel, which reports the five (R5), eight (R8), and six (R6) data points, as no $\sigma_{\mathrm{v}}$ data is available for NGC 5986).

Charbonnel \& Chantereau 2016). First, Charbonnel et al. (2013) and Charbonnel \& Chantereau (2016) showed that the maximum $\mathrm{Na}$ abundance expected on the AGB for a given age and metallicity strongly depends on the mass-loss rate adopted on the RGB (see also Cassisi et al. 2014), especially for the oldest GCs and the most metal-rich ones. This is clear from Fig. 11 where we show the predictions for the ratio of the $\mathrm{Na}$ spreads on the AGB and RGB for two values (0.5 and $0.65)$ of the $\eta$ parameter adopted in Reimers prescription used

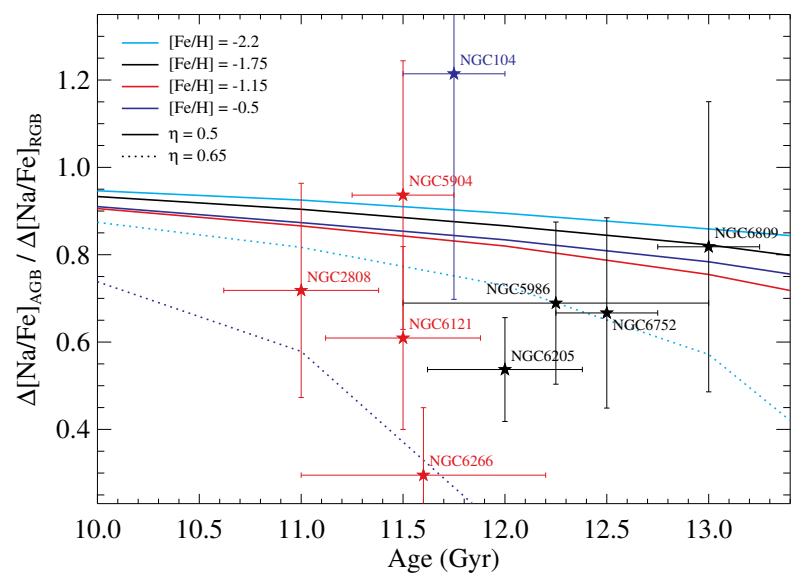

Fig. 11. Ratio of the $[\mathrm{Na} / \mathrm{Fe}]$ spreads on the $\mathrm{AGB}$ and RGB as a function of GC age. Predictions of Charbonnel \& Chantereau (2016) for the original FRMS scenario are shown for different values of metallicity (colors) and for two values of the $\eta$ parameter adopted for Reimers mass loss along the RGB ( 0.5 and 0.65 , solid and dotted lines respectively). The values derived in the present analysis are shown for the nine GCs (blue, red, and black stars, with the colors of the symbols indicating the theoretical track that has the closest $[\mathrm{Fe} / \mathrm{H}]$ from that of each individual GC).

by Charbonnel \& Chantereau (2016) to compute the mass-loss rate on the RGB. The trends with age and metallicity become stronger when higher mass-loss rates are considered. Additionally, the comparison between the predictions and the observed data for the nine GCs plotted in Fig. 11 supports variations of the RGB mass-loss efficiency from one GC to another within the range derived for $\eta$ by McDonald \& Zijlstra (2015, median and maximum values of 0.477 and 0.65 respectively) to explain the variations of the horizontal branch morphology for 56 Galactic GCs.

Second, both the observed and theoretical trends with age and metallicity are very weak (and we showed that in the observational case they depend on the considered subsamples), and scatters and exceptions exist in the data as can be seen in Fig. 10. This indicates to some extent that the fraction of 2P AGB stars is not simply affected by one single parameter/factor. Mass loss is certainly an important player as discussed above.

Third, although the ages we use here are taken from a single source (except for NGC 6266), they were not derived for the $[\mathrm{Fe} / \mathrm{H}]$ values reported in the present paper, which might also introduce some confusion in the derivation of the trends with age and metallicity.

Finally, the initial $\mathrm{Na}-\mathrm{He}$ correlation and its dependency with cluster properties is an important parameter to predict the theoretical value of the maximum $\mathrm{Na}$ expected on the AGB for a given GC. Unfortunately, this correlation is not constrained yet, which forced Charbonnel \& Chantereau (2016) to assume a similar relation over the metallicity range they investigated. To date, the only GCs for which non-negligible He enrichment were estimated using both photometry and spectroscopic measurements are NGC 2808 (Milone et al. 2015b) and NGC 6266 (Milone 2015). However, their Na distributions on the AGB are radically different and more similar to other GCs (NGC 6121 and NGC 6752, respectively) for which very modest He variations have been estimated (Villanova et al. 2012, for NGC 6121; Milone et al. 2013, for NGC 6752; and Nardiello et al. 2015a, for both GCs). Therefore, the theoretical interpretation of the data is not straightforward, bearing also in mind that the 
above-mentioned comparisons depend on the chosen abundance data set (cf. abundance discrepencies between different studies mentioned earlier on).

Overall, and as already pointed out in a more general context by Bastian et al. (2015), the broad variety of chemical patterns certainly reveals a high degree of stochasticity that is challenging our understanding of the formation and evolution of GCs and of their stellar populations.

\section{Summary}

After the claim by C13 that no Na-rich AGB stars were present in NGC 6752, several studies were recently devoted to the determination of the Na content of AGB stars in GCs. In this work, we present the first analysis for NGC 6809, together with that of a large stellar sample in NGC 104 and NGC 6121, and a reanalysis of NGC 6752. This is complementary to our previous study of NGC 2808 (Paper I). For these five GCs, we use a photometric method to derive effective temperature and surface gravity of the sample stars. Equivalent widths of unblended iron lines are measured to determine $[\mathrm{Fe} / \mathrm{H}]$, and the $\mathrm{Na}$ abundances are derived via spectrum synthesis, assuming local thermodynamic equilibrium (LTE). Non-LTE corrections are then applied to both $\mathrm{Fe} \mathrm{I}$ and $\mathrm{Na}$ abundances, following the prescriptions by Lind et al. (2011), Bergemann et al. (2012), and Lind et al. (2012). We provide a large set of stellar parameters and $\mathrm{Na}$ and $\mathrm{Fe}$ abundances derived self-consistently for the sample of 254 RGB stars and 145 AGB stars in five GCs. We compare our results with those of the literature, and we include in our discussion the only four other GCs (NGC 5904, NGC 5986, NGC 6205, and NGC 6266) for which Na has been studied along the AGB. The total sample of nine clusters covers a large range of $[\mathrm{Fe} / \mathrm{H}]$ $(-1.86$ to -0.82$)$, age (11 to $13 \mathrm{Gyr})$, and global properties.

We chose to use the $[\mathrm{Na} / \mathrm{H}]_{\mathrm{NLTE}}$ data to avoid the influence of the FeI-FeII discrepancy that has different behaviors in RGB and AGB stars, thus hampering the analysis. In addition, we define as Na-rich stars, or $2 \mathrm{P}$ stars, the objects that have a $[\mathrm{Na} / \mathrm{H}]$ value above $[\mathrm{Na} / \mathrm{H}]_{\text {cri }}=[\mathrm{Na} / \mathrm{H}]_{\min }+0.3 \mathrm{dex}$, where $[\mathrm{Na} / \mathrm{H}]_{\min }$ is the minimum Na value derived for the sample ( $\mathrm{RGB}+\mathrm{AGB})$ in a given cluster and 0.3 dex is about one third of the $[\mathrm{Na} / \mathrm{H}]$ spread. This definition agrees well with the general concept that 2P GC stars have higher $\mathrm{Na}$ abundance than field stars of similar metallicity. Although different criteria as well as abundance indicators (e.g., $[\mathrm{Na} / \mathrm{Fe}]$ combined with $\mathrm{O}$ abundances, Lapenna et al. 2016) can obviously lead to different evaluations of the ratios between $1 \mathrm{P}$ and $2 \mathrm{P}$ stars, we chose this approach to carry out a consistent analysis of the $\mathrm{Na}$ abundance for our large sample of RGB and AGB stars in the nine GCs.

Our analysis confirms the complex picture of the Na abundances in the AGB stellar populations in Galactic GCs. Eight out of nine GCs under scrutiny exhibit a lower $[\mathrm{Na} / \mathrm{H}]$ spread on the AGB than on the RGB, with the maximum $[\mathrm{Na} / \mathrm{H}]$ abundance being consistently lower on the AGB than on the RGB. The only exception is the most metal-rich cluster NGC 104, whose AGB stars show slightly larger Na spread than its RGB ones. Two clusters, NGC 6752 and NGC 6266, clearly stand out by showing (almost) no Na-rich (2P) AGB stars among the sample stars analyzed so far. We note, however, that uncertainties still exist since $i$. different conclusions have been reported for NGC 6752 thus demanding further homogeneous studies in both AGB and RGB stars; and ii. no firm conclusion can be drawn for NGC 6266, due to the paucity of stars scrutinized so far. We then find all possible behaviors between these two extremes. In NGC 6121, NGC 5904, and NGC 2808, the AGB stars occupy about the bottom two thirds of the Na distribution of the RGB stars, with a difference of $0.26,0.25$, and 0.21 dex respectively between the maximum $[\mathrm{Na} / \mathrm{H}]$ value of the $\mathrm{AGB}$ and $\mathrm{RGB}$ samples, indicating a deficit of very Na-rich AGB stars in these clusters. In the case of NGC 5986, instead, the AGB and RGB samples show comparable spreads in $[\mathrm{Na} / \mathrm{H}]$ with the maximum $\mathrm{Na}$ abundance of AGB stars being 0.14 dex lower than their RGB counterparts, but the AGB sample is still rather limited, thus weakening any drawn conclusion. In NGC 6809 the Na abundance of the RGB sample spreads more evenly while the AGB stars tend to be more concentrated, and a 0.12 dex lower $[\mathrm{Na} / \mathrm{H}]_{\max }$ present in the AGB sample compared to that of the RGB one. Finally, NGC 6205 seems to have very few $1 \mathrm{P}$ AGB stars, and the maximum $\mathrm{Na}$ values for its RGB and AGB components are marginally consistent within the observational errors.

Linear fits between the fraction of Na-rich 2P AGB stars and the GC parameters reveal that the AGB $2 \mathrm{P}$ fraction slightly anticorrelates with GC central concentration, with no conclusive results on possible trends with other GC parameters since they depend on the considered subsamples. By checking the AGB populations of pairs/subgroups of GCs and the trend of AGB Na abundance distributions of the nine GCs, we find that the current data roughly support the theoretical prediction of the original FRMS scenario according to which the initial $\mathrm{Na}$ and $\mathrm{He}$ abundances were correlated in the original mixture of the GC stars we observe today. However and as underlined in the discussion, this cannot be considered as a strong conclusion. Indeed, the predictions for the evolution of the stellar models along the AGB strongly depend on the mass loss on the RGB, and the initial $\mathrm{Na}-\mathrm{He}$ correlation and its possible dependency with cluster properties is not sufficiently constrained yet. The fact that both the observed and the theoretical trends with age, metallicity, and other global GC properties are mostly very weak, and that scatter and exceptions exist indicate that the fraction of $2 \mathrm{P}$ AGB stars is affected by more than one or two factors and is probably subject to stochasticity.

Acknowledgements. Y.W. acknowledges the support from the European Southern Observatory, via its ESO Studentship programme. This work was partly funded by the National Natural Science Foundation of China under grants 1233004 and 11390371, as well as the Strategic Priority Research Program The Emergence of Cosmological Structures of the Chinese Academy of Sciences, Grant No. XDB09000000. C.C. and W.C. acknowledge support from the Swiss National Science Foundation (FNS) for the project 200020-159543 Multiple stellar populations in massive star clusters - Formation, evolution, dynamics, impact on galactic evolution. We are indebted to Peter Stetson for kindly providing us with accurate Johnson-Morgan photometry. We thank Simon Campbell and Karin Lind for useful discussions, the latter also for giving us access to her NLTE correction grids. We thank the International Space Science Institute (ISSI, Bern, $\mathrm{CH}$ ) for welcoming the activities of ISSI Team 271 Massive star clusters across the Hubble Time (2013-2016). Finally, we thank the anonymous referees for their useful comments. This work has made use of the VALD database, operated at Uppsala University, the Institute of Astronomy RAS in Moscow, and the University of Vienna.

\section{References}

Alonso, A., Arribas, S., \& Martínez-Roger, C. 1999, A\&AS, 140, 261

Anderson, J., Piotto, G., King, I. R., Bedin, L. R., \& Guhathakurta, P. 2009, ApJ, 697, L58

Asplund, M., Grevesse, N., Sauval, A. J., \& Scott, P. 2009, ARA\&A, 47, 481

Bastian, N., \& Lardo, C. 2015, MNRAS, 453, 357

Bastian, N., Lamers, H. J. G. L. M., de Mink, S. E., et al. 2013, MNRAS, 436, 2398

Bastian, N., Cabrera-Ziri, I., \& Salaris, M. 2015, MNRAS, 449, 3333

Bergemann, M., Lind, K., Collet, R., Magic, Z., \& Asplund, M. 2012, MNRAS, 427, 27

Bonatto, C., Campos, F., \& Kepler, S. O. 2013, MNRAS, 435, 263 
Bono, G., Stetson, P. B., Sanna, N., et al. 2008, ApJ, 686, L87

Boyles, J., Lorimer, D. R., Turk, P. J., et al. 2011, ApJ, 742, 51

Campbell, S. W., D’Orazi, V., Yong, D., et al. 2013, Nature, 498, 198

Campbell, S. W., MacLean, B. T., D’Orazi, V., et al. 2017, A\&A, 605, A98

Cardelli, J. A., Clayton, G. C., \& Mathis, J. S. 1989, ApJ, 345, 245

Carretta, E. 2013, A\&A, 557, A128

Carretta, E. 2014, ApJ, 795, L28

Carretta, E. 2016, ArXiv e-prints [arXiv: 1611.04728]

Carretta, E., Gratton, R. G., Lucatello, S., Bragaglia, A., \& Bonifacio, P. 2005, A\&A, 433, 597

Carretta, E., Bragaglia, A., Gratton, R. G., et al. 2006, A\&A, 450, 523

Carretta, E., Bragaglia, A., Gratton, R. G., et al. 2009, A\&A, 505, 117

Carretta, E., Bragaglia, A., Gratton, R. G., et al. 2010, A\&A, 516, A55

Carretta, E., Bragaglia, A., Gratton, R. G., et al. 2014, A\&A, 564, A60

Cassisi, S., \& Salaris, M. 2014, A\&A, 563, A10

Cassisi, S., Salaris, M., Pietrinferni, A., Vink, J. S., \& Monelli, M. 2014, A\&A, 571, A81

Chantereau, W., Charbonnel, C., \& Decressin, T. 2015, A\&A, 578, A117

Chantereau, W., Charbonnel, C., \& Meynet, G. 2016, A\&A, 592, A111

Charbonnel, C. 2016, in EAS Pub. Ser., 80, eds. E. Moraux, Y. Lebreton, \& C. Charbonnel, 177

Charbonnel, C., \& Chantereau, W. 2016, A\&A, 586, A21

Charbonnel, C., Chantereau, W., Decressin, T., Meynet, G., \& Schaerer, D. 2013 A\&A, 557, L17

Charbonnel, C., Chantereau, W., Krause, M., Primas, F., \& Wang, Y. 2014, A\&A, 569, L6

Cordero, M. J., Pilachowski, C. A., Johnson, C. I., et al. 2014, ApJ, 780, 94

Cudworth, K. M., \& Rees, R. 1990, AJ, 99, 1491

D’Antona, F., Ventura, P., Caloi, V., et al. 2010, ApJ, 715, L63

de Mink, S. E., Pols, O. R., Langer, N., \& Izzard, R. G. 2009, A\&A, 507, L1

Decressin, T., Charbonnel, C., \& Meynet, G. 2007a, A\&A, 475, 859

Decressin, T., Meynet, G., Charbonnel, C., Prantzos, N., \& Ekström, S. 2007b, A\&A, 464, 1029

Decressin, T., Baumgardt, H., Charbonnel, C., \& Kroupa, P. 2010, A\&A, 516 A73

Denissenkov, P. A., \& Hartwick, F. D. A. 2014, MNRAS, 437, L21

Denissenkov, P. A., VandenBerg, D. A., Hartwick, F. D. A., et al. 2015, MNRAS, 448,3314

D’Ercole, A., D’Antona, F., Ventura, P., Vesperini, E., \& McMillan, S. L. W. 2010, MNRAS, 407, 854

di Criscienzo, M., Ventura, P., D’Antona, F., Milone, A., \& Piotto, G. 2010, MNRAS, 408, 999

García-Hernández, D. A., Mészáros, S., Monelli, M., et al. 2015, ApJ, 815, L4

Gratton, R. G., Carretta, E., Eriksson, K., \& Gustafsson, B. 1999, A\&A, 350, 955

Gratton, R. G., Bragaglia, A., Carretta, E., et al. 2003, A\&A, 408, 529

Gruyters, P., Casagrande, L., Milone, A. P., et al. 2017, A\&A, 603, A37

Gustafsson, B., Edvardsson, B., Eriksson, K., et al. 2008, A\&A, 486, 951

Harris, W. E. 1996, AJ, 112, 1487

Hendricks, B., Stetson, P. B., VandenBerg, D. A., \& Dall'Ora, M. 2012, AJ, 144 25

Ivans, I. I., Kraft, R. P., Sneden, C., et al. 2001, AJ, 122, 1438

Izzard, R. G., de Mink, S. E., Pols, O. R., et al. 2013, Mem. Soc. Astron. It., 84 171

Johnson, C. I., \& Pilachowski, C. A. 2012, ApJ, 754, L38

Johnson, C. I., McDonald, I., Pilachowski, C. A., et al. 2015, AJ, 149, 71

Johnson, C. I., Caldwell, N., Rich, R. M., et al. 2017, ApJ, 842, 24

Khalaj, P., \& Baumgardt, H. 2015, MNRAS, 452, 924

Krause, M., Charbonnel, C., Decressin, T., Meynet, G., \& Prantzos, N. 2013, A\&A, 552, A121
Krause, M. G. H., Charbonnel, C., Bastian, N., \& Diehl, R. 2016, A\&A, 587, A53

Lai, D. K., Smith, G. H., Bolte, M., et al. 2011, AJ, 141, 62

Lapenna, E., Mucciarelli, A., Lanzoni, B., et al. 2014, ApJ, 797, 124

Lapenna, E., Mucciarelli, A., Ferraro, F. R., et al. 2015, ApJ, 813, 97

Lapenna, E., Lardo, C., Mucciarelli, A., et al. 2016, ApJ, 826, L1

Lardo, C., Salaris, M., Savino, A., et al. 2017, MNRAS, 466, 3507

Larsen, S. S., Brodie, J. P., Forbes, D. A., \& Strader, J. 2014, A\&A, 565, A98

Larsen, S. S., Baumgardt, H., Bastian, N., et al. 2015, ApJ, 804, 71

Lind, K., Asplund, M., Barklem, P. S., \& Belyaev, A. K. 2011, A\&A, 528, A103

Lind, K., Bergemann, M., \& Asplund, M. 2012, MNRAS, 427, 50

Mackey, A. D., \& van den Bergh, S. 2005, MNRAS, 360, 631

MacLean, B. T., Campbell, S. W., De Silva, G. M., et al. 2016, MNRAS, 460 , L69

Maeder, A., \& Meynet, G. 2006, A\&A, 448, L37

Marconi, M., Coppola, G., Bono, G., et al. 2015, ApJ, 808, 50

Marino, A. F., Villanova, S., Piotto, G., et al. 2008, A\&A, 490, 625

Marino, A. F., Milone, A. P., Przybilla, N., et al. 2014, MNRAS, 437, 1609

Marino, A. F., Milone, A. P., Yong, D., et al. 2017, ApJ, 843, 66

McDonald, I., \& Zijlstra, A. A. 2015, MNRAS, 448, 502

Milone, A. P. 2015, MNRAS, 446, 1672

Milone, A. P., Piotto, G., Bedin, L. R., et al. 2012a, A\&A, 537, A77

Milone, A. P., Piotto, G., Bedin, L. R., et al. 2012b, ApJ, 744, 58

Milone, A. P., Marino, A. F., Piotto, G., et al. 2013, ApJ, 767, 120

Milone, A. P., Marino, A. F., Piotto, G., et al. 2015a, MNRAS, 447, 927

Milone, A. P., Marino, A. F., Piotto, G., et al. 2015b, ApJ, 808, 51

Monelli, M., Milone, A. P., Stetson, P. B., et al. 2013, MNRAS, 431, 2126

Mucciarelli, A., Salaris, M., Lovisi, L., et al. 2011, MNRAS, 412, 81

Mucciarelli, A., Lapenna, E., Massari, D., Ferraro, F. R., \& Lanzoni, B. 2015, ApJ, 801, 69

Nardiello, D., Milone, A. P., Piotto, G., et al. 2015a, A\&A, 573, A70

Nardiello, D., Piotto, G., Milone, A. P., et al. 2015b, MNRAS, 451, 312

Olech, A., Kaluzny, J., Thompson, I. B., et al. 1999, AJ, 118, 442

Pancino, E., Rejkuba, M., Zoccali, M., \& Carrera, R. 2010, A\&A, 524, A44

Pasquini, L., Alonso, J., Avila, G., et al. 2003, in Instrument Design and Performance for Optical/Infrared Ground-based Telescopes, eds. M. Iye, \& A. F. M. Moorwood, SPIE Conf. Ser., 4841, 1682

Pasquini, L., Mauas, P., Käufl, H. U., \& Cacciari, C. 2011, A\&A, 531, A35

Piotto, G., Milone, A. P., Anderson, J., et al. 2012, ApJ, 760, 39

Piotto, G., Milone, A. P., Marino, A. F., et al. 2013, ApJ, 775, 15

Piotto, G., Milone, A. P., Bedin, L. R., et al. 2015, AJ, 149, 91

Prantzos, N., \& Charbonnel, C. 2006, A\&A, 458, 135

Prantzos, N., Charbonnel, C., \& Iliadis, C. 2007, A\&A, 470, 179

Ramírez, I., \& Meléndez, J. 2005, ApJ, 626, 465

Renzini, A., D'Antona, F., Cassisi, S., et al. 2015, MNRAS, 454, 4197

Richter, P., Hilker, M., \& Richtler, T. 1999, A\&A, 350, 476

Roediger, J. C., Courteau, S., Graves, G., \& Schiavon, R. P. 2014, ApJS, 210, 10

Schaerer, D., \& Charbonnel, C. 2011, MNRAS, 413, 2297

Sills, A., \& Glebbeek, E. 2010, MNRAS, 407, 277

Skrutskie, M. F., Cutri, R. M., Stiening, R., et al. 2006, AJ, 131, 1163

Sneden, C. A. 1973, Ph.D. Thesis, The University of Texas at Austin

Stetson, P. B. 2000, PASP, 112, 925

Stetson, P. B. 2005, PASP, 117, 563

VandenBerg, D. A., Brogaard, K., Leaman, R., \& Casagrande, L. 2013, ApJ, 775, 134

Ventura, P., \& D'Antona, F. 2011, MNRAS, 410, 2760

Ventura, P., D'Antona, F., Mazzitelli, I., \& Gratton, R. 2001, ApJ, 550, L65

Ventura, P., Di Criscienzo, M., Carini, R., \& D'Antona, F. 2013, MNRAS, 431, 3642

Villanova, S., Geisler, D., Piotto, G., \& Gratton, R. G. 2012, ApJ, 748, 62 